\title{
Reconstruction of quantum theory on the basis of the formula of total probability
}

\author{
Andrei Khrennikov \\ International Center for Mathematical Modeling in Physics, Engineering and Cognitive science MSI, Växjö \\ University, S-35195, Sweden
}

\begin{abstract}
The notion of context (complex of physical conditions) is basic in this paper. We show that the main structures of quantum theory (interference of probabilities, Born's rule, complex probabilistic amplitudes, Hilbert state space, representation of observables by operators) are present in a latent form in the classical Kolmogorov probability model. However, this model should be considered as a calculus of contextual probabilities. In our approach it is forbidden to consider abstract context independent probabilities: "first context and then probability." We start with the conventional formula of total probability for contextual (conditional) probabilities and then we rewrite it by eliminating combinations of incompatible contexts from consideration. In this way we obtain interference of probabilities without to appeal to the Hilbert space formalism or wave mechanics. However, we did not just reconstruct the probabilistic formalism of conventional quantum mechanics. Our contextual probabilistic model is essentially more general and, besides the projection to the complex Hilbert space, it has other projections. The most important new prediction is the possibility (at least theoretical) of appearance of hyperbolic interference. A projection of the classical contextual probabilistic model to the hyperbolic Hilbert space (a module over the commutative two dimensional Clifford algebra) has some similarities with the projection to the complex Hilbert space. However, in the hyperbolic quantum mechanics the principle of superposition is violated. Our realistic (but contextual!) approach to quantum mechanics does not contradict to various "no-go theorems", e.g., von Neumann, Bell, Kochen-Specker. We emphasize that our projection of the classical probabilistic model to the complex Hilbert space is based on two incompatible observables ("reference observables"), e.g., the position and the momentum, or the position and the energy. Only these two observables can be considered as objective properties of quantum systems.
\end{abstract}

\section{INTRODUCTION}

It is well know that the classical Kolmogorov probabilistic model [1] differs crucially from the quantum probabilistic model, see, e.g., [2]-[37] for details and debates. The classical model is based on a set-theoretical structure ( $\sigma$-field of subsets of some set $\Omega$ ); there is no need in using complex numbers; physical observables are represented by functions on $\Omega$ and there is no need in using noncommutative structures. The quantum model is based on a complex Hilbert space. The appearance of complex numbers in the model with real-valued probabilities and physical observables is one of quantum mysteries. Instead of probability distributions, there are considered complex probabilistic amplitudes (or in the abstract approach normalized vectors in the Hilbert state space). The probabilistic interpretation of such amplitudes (or vectors in the Hilbert space) is given by Born's rule. It is hard to find probabilistic roots of this rule in the conventional quantum theory. ${ }^{1}$ By using the Hilbert space formalism or Schrödinger's wave mechanics it is possible to get interference of probabilities (which is also observed in many quantum experiments, e.g., the two slit experiment). There is no reasonable explanation of interference. ${ }^{2}$ Physical observables are represented by self-adjoint operators. Operators are noncommutative for incompatible observables. There is no explanation of the appearance of the noncommutative structure in the theory with real-valued (so commuting) physical observables.

\footnotetext{
${ }^{1}$ It is clear that M. Born wanted to modify Schrödinger's idea that the wave function gives the distribution of the electric charge of electron in space. First time Born's rule appeared as a footnote and in the first variant of the Born's paper there was proposed to consider not square, but the absolute value of $\psi$.

${ }^{2}$ Self-interference of an individual particle is a metaphor. As was pointed out by N. Bohr, there is no way to combine the corpuscular model and interference of probabilities. This is the essence of the principle of complementarity. But we emphasize that this principle is a consequence of one special interpretation of quantum mechanics - the Copenhagen interpretation.
} 
These probabilistic differences between classical and quantum probabilistic models induce a rather mystical viewpoint to properties of quantum systems. In this paper we shall see that the gap between classical and quantum probabilistic models is not so huge as it was commonly believed, see [2]-[37]. The quantum probabilistic model can be considered as a projection of the classical model to the complex Hilbert space, see [38]-[45]. As any projection, the quantum projection does not provide the complete image of the prequantum classical probabilistic model. In particular, there can be constructed another projection - to a so called hyperbolic Hilbert space, [38]-[45].

The notion of context (complex of physical conditions) is basic in this paper. We show that the main structures of quantum theory (interference of probabilities, Born's rule, complex probabilistic amplitudes, Hilbert state space, representation of observables by operators) are present in a latent form in the classical Kolmogorov probability space. ${ }^{3}$ However, this space should be considered as the basis of a calculus of contextual probabilities. In our approach it is forbidden to consider abstract context independent probabilities: "first context and then probability." We start with the conventional formula of total probability for contextual (conditional) probabilities and then we rewrite it by eliminating combinations of incompatible contexts from consideration. ${ }^{4}$ In this way we obtain interference of probabilities without to appeal to the Hilbert space formalism or wave mechanics. By starting with the formula of total probability with interference term (under some restriction on the magnitude of this term) we represent contexts belonging to a special class (so called trigonometric contexts) by complex probabilistic amplitudes. The Born's rule immediately appears in such a representation. In our contextual model this rule is just a special form of writing the formula of total probability with the interference term.

As was already mentioned, we did not only reconstruct the probabilistic formalism of conventional quantum mechanics. Our contextual probabilistic model is essentially more general and, besides the projection to the complex Hilbert space, it has other projections. The most important new prediction is the possibility (at least theoretical) of appearance of hyperbolic interference. For the conventional trigonometric interference the brightness of interference picture is changed as a trigonometric function, e.g., cos. For the hyperbolic interference the brightness of interference picture is changed as a hyperbolic function, e.g., cosh, so exponentially. It may occur that such an interference with exponentially varied brightness would be found in future experiments.

Our realistic (but contextual!) approach to quantum mechanics does not contradict to various "no-go theorems", e.g., von Neumann, Kochen-Specker, Bell. We would like to pay attention that all business with "no-go theorems" is about the correspondence between two mathematical probabilistic models: $M_{\mathrm{cl}}$ and $M_{\text {quant }}$. The main problem in the no-go activity is that nobody paid attention to the evident fact that to study such a mathematical problem, it is not enough to describe two mathematical models. One should also fix a class of rules of the correspondence between those models. In fact, in each "no-go theorem" there is fixed such a class of rules. And "no-go" means only no-go for such a class of rules. Classes of rules for classical-quantum correspondence can be considered as classes of maps from $M_{\mathrm{cl}} \rightarrow M_{\text {quant }}$ or $M_{\text {quant }} \rightarrow M_{\mathrm{cl}}$. However, any mathematician understands well that if one proved that there does not exist a map of some class which maps, e.g., $M_{\mathrm{cl}}$ into (or onto) $M_{\text {quant }}$, this does not mean that there could not be found a map of another class. ${ }^{5}$

We emphasize that our projection of the classical probabilistic model to the complex Hilbert space is based on two fixed incompatible observables ("reference observables"), e.g., the position and the momentum, or the position and the energy. Only these two observables can be considered as objective properties of quantum systems. In our model these observables are realized by classical incompatible random variables and incompatibility is defined in purely classical probabilistic framework, see Definition 2. Let us fix some pair of reference observables $a$ and $b$ taking values in the field of real numbers $\mathbf{R}$. Let $C$ be some trigonometric context (i.e., a complex of physical conditions inducing the ordinary cos-interference). By using $C$-contextual probabilities for $a$ and $b$ we represent the context $C$ by a complex probability amplitude $\psi_{C}$ (this amplitude is, in fact, encoded in the formula of total probability with the interference

\footnotetext{
${ }^{3}$ Hence, it would be better to speak not about hidden variables for quantum theory, but about hidden presence of quantum structures in the classical probability space.

${ }^{4}$ Let us imagine that in 19th (or even in 18th) century a probabilist would like to modify the formula of total probability in such a way that "intersections of incompatible conditions" would be eliminated. In such a way he should automatically come to our formula of total probability with the interference term. Unfortunately, this did not happen. Interference of probabilities was discovered not in pure mathematics, but in experimental physics. Then it was derived by using the quantum formalism.

5 Let us illustrate the situation with classical-quantum correspondence by the following example. Let one choose the class of diffeomorphisms (i.e., one-to-one $C^{1}$-maps with inverse $C^{1}$-maps) as the class of correspondence-maps for configuration spaces of dynamical systems. Let he proved that two configuration spaces are not diffeomorphic, i.e., it is impossible to construct a diffeomorphism between these spaces. But anybody understands that such a result does not mean that it is impossible to construct, e.g., a homeomorphism (i.e., one-to-one continuous map with inverse continuous) between these spaces.
} 
term, see (13)). This representation induces a representation of the reference observables $a$ and $b$ by self-adjoint operators $\hat{a}$ and $\hat{b}$. Incompatibility of random variables $a$ and $b$ implies that these operators do not commute: $[\hat{a}, \hat{b}] \neq 0$.

The crucial point in understanding why our contextual model does not contradict to "no-go theorem" of von Neumann is that the algebraic structure on the set of values of random variables, the field of real numbers $\mathbf{R}$, is not consistent with the algebraic structure on the space linear operators in the (complex) Hilbert state space. For example, we shall see that in general the image of the random variable $d(\omega)=a(\omega)+b(\omega)$ is not given by the operator $\hat{d}=\hat{a}+\hat{b}$. But the correspondence $d(\omega) \rightarrow \hat{d}$ is one of the conditions of the von Neumann "no-go theorem." This condition was criticized by many authors, see [13], [16]. Therefore it is not so surprising that it is violated in our model. There exist contexts $C$ such that $d(\omega)$ and $\hat{d}$ have different probability distributions (with respect to the context $C$ and the corresponding state $\psi_{C}$, respectively). Surprisingly, in spite of difference of probability distributions, classical and quantum averages coincide: $E(d / C)=\left(\hat{d} \psi_{C}, \psi_{C}\right)$. The same is valid for any random variable of the form $d(\omega)=f(a(\omega))+g(b(\omega))$ (and the corresponding quantum observable $\hat{d}=f(\hat{a})+g(\hat{b})$ ). Thus in our model for a wide class of "realistic" random variables (in particular, for any "energy variable" $\mathscr{H}(\omega)=\frac{a^{2}(\omega)}{2 m}+V(b(\omega))$ and the corresponding "Hamiltonian" $\left.\hat{\mathscr{H}}=\frac{\hat{a^{2}}}{2 m}+V(\hat{b})\right)$ quantum averages coincides with prequantum classical averages (so, in particular, $\left.E(\mathscr{H} / C)=\left(\hat{\mathscr{H}} \psi_{C}, \psi_{C}\right)\right)$.

The existence of our realistic prequantum model does not contradict to "no-go theorem" of Kochen-Specker, since our model does not define a one-to-one map from the space of quantum observables into the space of classical random variables. In this paper we do not consider composite systems. Therefore we do not discuss relations with Bell's theorem.

\section{INTERFERENCE OF PROBABILITIES}

\section{The conventional Kolmogorov probabilistic model}

Let $\mathscr{K}=(\Omega, \mathscr{F}, \mathbf{P})$ be a Kolmogorov probability space, [1], [46]. This space is the basis of the classical probabilistic model, the Kolmogorov model [1], [46]. As any model of reality, the Kolmogorov model consists of two parts: the mathematical formalism and the interpretation.

Mathematical formalism. Here $\Omega$ is an arbitrary set, $\mathscr{F}$ is a $\sigma$-field ${ }^{6}$ of subsets of $\Omega ; \mathbf{P}$ is a probability measure on $\mathscr{F}$ : a countably-additive measure with values in $[0,1]$ such that $\mathbf{P}(\Omega)=1$.

Kolmogorov's interpretation. Points $\omega \in \Omega$ represent elementary events. Some special sets of elementary events represent events; it is supposed that the family of all events is a $\sigma$-field $\mathscr{F}$. For an event $A \in \mathscr{F}, \mathbf{P}(A)$ is the probability of occurrence of the event A. Observables (e.g., physical) are represented by random variables. We recall that a random variable is a measurable function $d: \Omega \rightarrow \mathbf{R}$ (so for any Borel subset $\Gamma$ of the field of real numbers $\mathbf{R}$, its preimage $D_{\Gamma}=\{\omega \in \Omega: d(\omega) \in \Gamma\}$ belongs to the $\sigma$-field $\left.\mathscr{F}\right)$. Conditional probability $\mathbf{P}(B / A)$ that an event $B$ occurs under the condition that an event $A$ has been occurred is defined by Bayes' formula:

$$
\mathbf{P}(A / C)=\frac{\mathbf{P}(A \cap C)}{\mathbf{P}(C)}, \mathbf{P}(C) \neq 0 .
$$

\section{The contextual Kolmogorov probabilistic model}

Here we use the same mathematical formalism as in the conventional Kolmogorov probabilistic model, the Kolmogorov probability space $\mathscr{K}=(\Omega, \mathscr{F}, \mathbf{P})$. However, structures of $\mathscr{K}$ have different interpretations.

Contextual interpretation of the Kolmogorov probability space. Points $\omega \in \Omega$ represent fundamental parameters of the model. ${ }^{7}$ Some special sets of fundamental parameters represent contexts - complexes of physical conditions. ${ }^{8}$

\footnotetext{
${ }^{6}$ A collection of subsets of $\Omega$ which contains $\Omega$ and the empty set $\emptyset$ and it is closed with respect to the operations of countable intersection and union of sets and it contains the complement to any its element.

7 We recall that we would not like to call $\omega$ hidden variables, since we are not looking for hidden parameters for the quantum model. We are looking for the hidden quantum structure in the Kolmogorov probability space $\mathscr{K}$.

${ }^{8}$ In this paper we consider only physical models. However, it is possible to use the same approach for, e.g., cognitive or psychological models, see [41], [47].
} 
It is supposed that sets representing contexts form a $\sigma$-field $\mathscr{F}$. In the opposite to the conventional Kolmogorov probabilistic model, $\mathbf{P}(C), C \in \mathscr{F}$, has no direct physical interpretation. In our model probability can be considered only as conditional (or better to say contextual) probability, see (2). As in the conventional Kolmogorov probabilistic model, observables are represented by random variables. For a random variable $d$, the conditional (contextual) probability $\mathbf{P}(d \in \Gamma / C), C \in \mathscr{F}$, is defined by the Bayes' formula:

$$
\mathbf{P}(d \in \Gamma / C)=\frac{\mathbf{P}\left(D_{\Gamma} \cap C\right)}{\mathbf{P}(C)}, \mathbf{P}(C) \neq 0 .
$$

In our model the Bayes' formula has the following meaning. To find the probability that a random variable $d \in \Gamma$ under the context $C$, there should be selected parameters $\omega \in \Omega$ which belong the intersection of the sets $D_{\Gamma}$ and $C$. These are all parameters $\omega \in C$ for that $d(\omega) \in \Gamma$. We remark that the Bayes' formula (2) gives the definition of probability in terms of the contextual Kolmogorov model. The "experimental probability" $\mathbf{P}_{\exp }(d \in \Gamma / C)$ is defined as the limit of the frequencies $v_{N}(d \in \Gamma / C)$ to find $d \in \Gamma$ in a series of $N$ observations under the complex of physical conditions $C .{ }^{9}$

The "experimental probability" $\mathbf{P}_{\exp }(d \in \Gamma / C)$ coincides with $\mathbf{P}(d \in \Gamma / C)$ as a consequence of the law of large numbers (if trails are independent). We have the same situation in the conventional Kolmogorov model.

In particular, if $d$ is a discrete random variable then

$$
\mathbf{P}(d=z \in \Gamma / C)=\frac{\mathbf{P}\left(D_{z} \cap C\right)}{\mathbf{P}(C)}, \mathbf{P}(C) \neq 0,
$$

where $D_{z}=\{\omega \in \Omega: d(\omega)=z\}$.

By our interpretation the set $D_{z}$ represents the context corresponding to filtration with respect to value $d=z$. We emphasize that that the operation of intersection of sets has nothing to do with with creating "intersections" of corresponding contexts. The probability $\mathbf{P}\left(D_{z} \cap C\right)$ has no physical interpretation by itself. ${ }^{10}$ We shall discuss this point in more detail in the next section.

\section{The formula of total probability}

As was remarked in introduction, our visualization of the latent quantum structure of the classical contextual probabilistic model is based on a contextual version of the well known formula of total probability. We start with recalling this formula in the conventional Kolmogorov approach.

Let $\mathscr{A}=\left\{A_{n}\right\}$ be finite or countable complete group of disjoint events ("partition of unity"):

$$
A_{i} \cap A_{j}=\emptyset, i \neq j, \quad \cup_{i} A_{i}=\Omega .
$$

Let $B, C \in \mathscr{F}$ be events and let $\mathbf{P}(C)>0$. We have the standard formula of total probability, see, e.g., [46]:

$$
\mathbf{P}(B / C)=\sum_{n} \mathbf{P}\left(A_{n} / C\right) \mathbf{P}\left(B / A_{n} \cap C\right)
$$

which can be easily derived:

$$
\mathbf{P}(B / C)=\frac{\mathbf{P}(B \cap C)}{\mathbf{P}(C)}=\sum_{n} \frac{\mathbf{P}\left(B \cap A_{n} \cap C\right) \mathbf{P}\left(A_{n} \cap C\right)}{\mathbf{P}(C) \mathbf{P}\left(A_{n} \cap C\right)} .
$$

This derivation was performed under the condition that

$$
\mathbf{P}\left(A_{n} \cap C\right)>0 \text { for all } n \text {. }
$$

\footnotetext{
${ }^{9}$ According to [48] there two levels of description of physical reality: ontic and epistemic. Kolmogorov probabilities $\mathbf{P}(d \in \Gamma / C)$ belong to the ontic level and frequency probabilities $\mathbf{P}_{\exp }(d \in \Gamma / C)$ belong to the epistemic level.

${ }^{10}$ We remark that any model of physical reality contains some mathematical structures which do not have direct physical interpretations. For example, A. N. Kolmogorov pointed out that the condition of countable-additivity do not permit physical verification, [1]. There can also exist measurable sets which do not correspond to physical events and so on.
} 
A contextual analog of this condition will play an important role in our theory. The formula (4) works well in all domains of science (it is the basis of Bayesian analysis), besides quantum physics (and may be psychology, see [41], [47]). In particular, let $a$ and $b$ be discrete random variables taking values $a \in Y=\left\{a_{i}, i=1, \ldots, k_{a}\right\}$ and $b \in X=\left\{b_{j}, j=1, \ldots, k_{b}\right\}$, where $k_{a}, k_{b}<\infty$. We have

$$
\mathbf{P}(b=x / C)=\sum_{y \in Y} \mathbf{P}(a=y / C) \mathbf{P}(b=x /(a=y) \cap C), x \in X .
$$

For further considerations it is useful to introduce sets:

$$
A_{y}=\{\omega \in \Omega: a(\omega)=y\}, y \in Y, \quad B_{x}=\{\omega \in \Omega: b(\omega)=x\}, x \in X .
$$

\section{The formula of total probability with interference term}

We now want to consider this formula in the contextual Kolmogorov model. Since the mathematical formalism is the same, there are no differences in mathematical calculations; the only difference is in the interpretation. Probabilities $\mathbf{P}(b=x / C), \mathbf{P}(a=y / C)$ are well defined from the contextual viewpoint. Let us now consider the probability $\mathbf{P}(b=x /(a=y) \cap C)=\mathbf{P}\left(b=x / A_{y} \cap C\right)$. Here considerations are not so straightforward. In the conventional Kolmogorov model the set $Q=(a=y) \cap C=A_{y} \cap C$ represents the event - the simultaneous occurrence of the events $A_{y}$ and $C$. To perform careful analysis of the contextual situation, at the moment we shall use different symbols for a context and the corresponding set in $\mathscr{F}$ representing this context: contexts will be denoted $\tilde{C}, \tilde{A_{y}}, \tilde{Q}, \ldots$ and corresponding sets $C, A_{y}, Q, \ldots$ In particular, here $\tilde{A_{y}}$ is the context of the $[a=y]$-filtration which is represented by the set $A_{y}$ in the Kolmogorov space.

In our model the set $Q=A_{y} \cap C$ represents some context $\tilde{Q}(y, C)$. But the representation of the set $Q$ in the form of the intersection of the sets $C$ and $A_{y}$ does not mean that the context $\tilde{Q}(C, y)$ is really the "intersection" of the contexts $\tilde{C}$ and $\tilde{A_{y}} .{ }^{11}$ The latter context, say $\tilde{M}(y, C)$ - "first we prepare an ensemble of systems under the complex of physical conditions $C$ and then perform the $a=y$ filtration" - need not be represented by the set $Q$. If the procedure of $a=y$ filtration disturbs the original context $C$, then there is no reason to assume that the context $\tilde{M}(y, C)$ should be represented by the set $Q=A_{y} \cap C$. So the first conclusion of our analysis is that in general the sets $Q=A_{y} \cap C$ in the right-hand side of (6) do not represent contexts $\tilde{M}(y, C)$. We remark that the contexts $\tilde{M}(y, C)$ can be easily designed experimentally and used for the collection of statistical data for frequency probabilities (which can be found in a long series of observations). What can we say about a context $\tilde{Q}$ which is represented by the set $Q$ ? In fact, not so much. This context should be created via the nondisturbative $[a=y]$-selection under the complex of physical conditions $C$. In general $\tilde{Q}$ cannot be constructed just through the combination of $\tilde{C}$ and $\tilde{A_{y}}$.

Since we do not know how to create the context $\tilde{Q}$, we would not be able to find corresponding experimental probabilities and the formula of total probability is not useful for applications (in spite of its validity in the underlying Kolmogorov model). ${ }^{12}$ Therefore it would be natural to try to exclude sets $Q=A_{y} \cap C$ from consideration and obtain a new variant of the formula of total probability. ${ }^{13}$ Our analysis of correspondence between creating new contexts and operations on sets representing contexts is finished. We shall again use the same symbol for a context and the set representing this context.

To simplify considerations, we shall consider only dichotomous random variables: $a \in Y=\left\{a_{1}, a_{2},\right\}, b \in X=$ $\left\{b_{1}, b_{2}\right\}$. Even this very simple model (the contextual Kolmogorov model with dichotomous observables) has (in a latent form) all distinguishing features of the quantum model.

Definition 1. (cf. (5) $A$ context $C \in \mathscr{F}$ is nondegenerate with respect to a random variable a if $\mathbf{P}\left(A_{y} \cap C\right) \neq 0$ for all $y \in Y$.

\footnotetext{
11 There is no such a postulate in our interpretation of the probability space $\mathscr{K}$.

12 By using terminology of [48] one can say that the formula of total probability is well defined on the ontic level of description of nature, but it could not be directly lifted to the epistemic level of description. We would like to modify this formula to get its analog which would be meaningful on both levels of description.

${ }^{13}$ We emphasize that we do not claim that the context $\tilde{Q}$ represented by $Q=A_{y} \cap C$ could not be created at all, cf. with Bohr's principle of complementarity and some interpretations of Heisenberg's uncertainty relations. We only observed that the conventional formula of total probability (6) contains sets $Q=A_{y} \cap C$ representing contexts $\tilde{Q}$ such that in general we do not know how to create them. Therefore we would like to exclude sets $Q=A_{y} \cap C$ from our consideration. Finally, we shall come to the same formalism that is used in quantum mechanics. But we shall escape a lot of quantum mysteries, since sets $Q=A_{y} \cap C$ are eliminated from formulas by a simple pragmatic reason.
} 
We denote the set of all $a$-nondegenerate contexts by the symbol $\mathscr{C}_{a}$.

Definition 2. Random variables $a$ and $b$ are called incompatible if $\mathbf{P}\left(A_{y} \cap B_{x}\right) \neq 0$ for all $y \in Y$ and $x \in X$.

Thus $a$ and $b$ are incompatible iff every $B_{x}$ is $a$-nondegerate and vice versa. We introduced incompatible random variables in purely classical framework (see appendix for some properties of such pairs of random variables). We shall see that this incompatibility contains (in a latent form) quantum incompatibility - representation by noncommutative operators.

Theorem 1. (Formula of total probability with interference term) Let $a$ and $b$ be incompatible random variables and let a context $C$ be a-nondegenerate. Then

$$
\mathbf{P}(b=x / C)=\sum_{y \in Y} \mathbf{P}(a=y / C) \mathbf{P}(b=x / a=y)+2 \lambda(b=x / a, C) \sqrt{\prod_{y \in Y} \mathbf{P}(a=y / C) \mathbf{P}(b=x / a=y)},
$$

where

$$
\lambda(b=x / a, C)=\frac{\delta(b=x / a, C)}{2 \sqrt{\mathbf{P}\left(a=a_{1} / C\right) \mathbf{P}\left(b=x / a=a_{1}\right) \mathbf{P}\left(a=a_{2} / C\right) \mathbf{P}\left(b=x / a=a_{2}\right)}}
$$

and

$$
\delta(b=x / a, C)=\mathbf{P}(b=x / C)-\sum_{y \in Y} \mathbf{P}(b=x / a=y) \mathbf{P}(a=y / C) .
$$

To prove Theorem we put expressions for $\lambda$ and $\delta$ into (7) and we obtain identity. In fact, (7) is just a representation of the probability $\mathbf{P}(b=x / C)$ in a special way. We choose the special representation of the perturbation $\delta(b=x / a, C)$ of $\sum_{y \in Y} \mathbf{P}(a=y / C) \mathbf{P}(b=x / a=y)$, namely its normalization by square root of all probabilities. At the beginning I expected that this normalization would produce quantities bounded by one. But in general it was not the case. We call $\lambda(b=x / a, C)$ coefficients of incompatibility. In our further investigations we will use the following result:

Lemma 1. Let conditions of Theorem 1 hold true. Then

$$
\sum_{x \in X} \delta(b=x / a, C)=0
$$

Proof. We have $1=\sum_{x \in X} \mathbf{P}(b=x / C)=\sum_{x \in X} \sum_{y \in Y} \mathbf{P}(a=y / C) \mathbf{P}(b=x / a=y)+\sum_{x \in X} \delta(b=x / a, C)$. But $\sum_{y \in Y}\left(\sum_{x \in X} \mathbf{P}(b=x / a=y)\right) \mathbf{P}(a=y / C)=1$.

As a consequence of this lemma we have:

$$
\sum_{x \in X} \lambda(b=x / a, C) \sqrt{\mathbf{P}\left(a=a_{1} / C\right) \mathbf{P}\left(a=a_{2} / C\right) \mathbf{P}\left(b=x / a=a_{1}\right) \mathbf{P}\left(b=x / a=a_{2}\right)}=0 .
$$

1). Suppose that both coefficients of incompatibility are relatively small

$$
|\lambda(b=x / a, C)| \leq 1, x \in X .
$$

In this case we can introduce new statistical parameters $\theta(b=x / a, C) \in[0,2 \pi]$ and represent the coefficients of statistical disturbance in the trigonometric form:

$$
\lambda(b=x / a, C)=\cos \theta(b=x / a, C) .
$$

Parameters $\theta(b=x / a, C)$ are said to be relative phases (or random variables $a$ and $b$.) This is purely probabilistic definition of phases. So we introduce geometry through probability.

In this case we obtain the following interference formula of total probability:

$$
\mathbf{P}(b=x / C)=\sum_{y \in Y} \mathbf{P}(a=y / C) \mathbf{P}(b=x / a=y)+2 \cos (b=x / a, C) \sqrt{\prod_{y \in Y} \mathbf{P}(a=y / C) \mathbf{P}(b=x / a=y)} .
$$

This is nothing other than the famous formula of interference of probabilities. ${ }^{14}$ Thus we found (hidden) interference of probabilities in the Kolmogorov probability space.

\footnotetext{
14 Typically this formula is derived by using the Hilbert space (unitary) transformation corresponding to the transition from one orthonormal basis to another and Born's probability postulate. The orthonormal basis under quantum consideration consist of eigenvectors of operators (noncommutative) corresponding to quantum physical observables $a$ and $b$.
} 
2). Suppose that both coefficients of incompatibility are relatively large

$$
|\lambda(b=x / a, C)| \geq 1, x \in X .
$$

In this case we can introduce new statistical parameters $\theta(b=x / a, C)) \in(-\infty,+\infty)$ and represent the coefficients of incompatibility in the hyperbolic form:

$$
\lambda(b=x / a, C)= \pm \cosh \theta(b=x / a, C) .
$$

Parameters $\theta(b=x / a, C)$ are said to be hyperbolic relative phases. In this case we obtain the following interference formula of total probability:

$$
\mathbf{P}(b=x / C)=\sum_{y \in Y} \mathbf{P}(a=y / C) \mathbf{P}(b=x / a=y) \pm 2 \cosh (b=x / a, C) \sqrt{\prod_{y \in Y} \mathbf{P}(a=y / C) \mathbf{P}(b=x / a=y)}
$$

We remark that in the ordinary formula for interference of probabilities (13) the expression in the right-hand side determines the quantity which belongs the segment $[0,1]$ for any angle $\theta$. In the hyperbolic case, see (15), this quantity belongs $[0,1]$ only for special range of angles $\theta$. But this is not a problem in our approach. We do not determine the probability $\mathbf{P}(b=x / C)$ through the right-hand side of (15). We proceed in the opposite way: the phase $\theta$ is determined through probabilities $\mathbf{P}(b=x / C), \mathbf{P}(a=y / C), \mathbf{P}(b=x / a=y)$. For some probabilities there exists the trigonometric representation, for other probabilities there exists the hyperbolic representation.

$3)$. Suppose that the absolute value of one of the coefficients $\lambda(b=x / a, C)$ is less than one and the absolute value of another coefficient is larger than one. Here we have the interference formula of total probability containing trigonometric as well as hyperbolic interference terms.

If incompatible random variables $a$ and $b$ are fixed, we shall often use the symbols $\delta(x / a, C), \lambda(x / a, C)$ instead of $\delta(b=x / a, C), \lambda(b=x / a, C)$.

\section{QUANTUM PROJECTION OF THE CLASSICAL MODEL}

Let us fix a pair of incompatible random variables $a=a_{1}, a_{2}, b=b_{1}, b_{2}$. We call such variables reference variables. For each pair $a, b$ of reference variables, we construct a projection of the contextual Kolmogorov model to the complex Hilbert space. We start from the trigonometric interference. We set

$$
\mathscr{C}^{\mathrm{tr}}=\left\{C \in \mathscr{C}_{a}:|\lambda(b=x / a, C)| \leq 1\right\}
$$

We call elements of $\mathscr{C}^{\text {tr }}$ trigonometric contexts. We shall see that quantum mechanics can be interpreted as a representation of trigonometric contexts. We shall also consider hyperbolic contexts which can be represented in a hyperbolic Hilbert space. In few further sections we shall consider only trigonometric contexts and in those sections we shall omit the upper index and use simply the symbol: $\mathscr{C} \equiv \mathscr{C}^{\mathrm{tr}}$.

\section{Interference and complex probability amplitude, Born's rule}

Let $C \in \mathscr{C}$. We set $p_{C}^{a}(y)=\mathbf{P}(a=y / C), p_{C}^{b}(x)=\mathbf{P}(b=x / C), p(x / y)=\mathbf{P}(b=x / a=y), x \in X, y \in Y$. The interference formula of total probability (13) can be written in the following form

$$
p_{C}^{b}(x)=\sum_{y \in Y} p_{C}^{a}(y) p(x / y)+2 \cos \theta_{C}(x) \sqrt{\Pi_{y \in Y} p_{C}^{a}(y) p(x / y)},
$$

where $\theta_{C}(x)=\theta(b=x / a, C)= \pm \arccos \lambda(b=x / a, C), x \in X, C \in \mathscr{C}$. Here

$$
\delta(b=x / a, C)=p_{c}^{b}(x)-\sum_{y \in Y} p_{C}^{a}(y) p(x / y) \text { and } \lambda(b=x / a, C)=\frac{\delta(b=x / a, C)}{2 \sqrt{\Pi_{y \in Y} p_{C}^{a}(y) p(x / y)}} .
$$

By using the elementary formula:

$$
D=A+B+2 \sqrt{A B} \cos \theta=\left|\sqrt{A}+e^{i \theta} \sqrt{B}\right|^{2},
$$


for $A, B>0, \theta \in[0,2 \pi]$, we can represent the probability $p_{C}^{b}(x)$ as the square of the complex amplitude (Born's rule):

$$
p_{C}^{b}(x)=\left|\psi_{C}(x)\right|^{2},
$$

where

$$
\psi(x) \equiv \psi_{C}(x)=\sqrt{p_{C}^{a}\left(a_{1}\right) p\left(x / a_{1}\right)}+e^{i \theta_{C}(x)} \sqrt{p_{C}^{a}\left(a_{2}\right) p\left(x / a_{2}\right)} .
$$

It is important to underline that since for each $x \in X$ phases $\theta_{C}(x)$ can be chosen in two ways (by choosing signs + or -) a representation of contexts by complex amplitudes is not uniquely defined. ${ }^{15}$

We denote the space of functions: $\psi: X \rightarrow \mathbf{C}$ by the symbol $E=\Phi(X, \mathbf{C})$. Since $X=\left\{b_{1}, b_{2}\right\}$, the $E$ is the two dimensional complex linear space. Dirac's $\delta$-functions $\left\{\delta\left(b_{1}-x\right), \delta\left(b_{2}-x\right)\right\}$ form the canonical basis in this space. We shall see that under a natural restriction on the matrices of transition probabilities:

$$
\psi_{B_{z}}(x)=\delta(z-x), z=b_{1}, b_{2} .
$$

For each $\psi \in E$ we have $\psi(x)=\psi\left(b_{1}\right) \delta\left(b_{1}-x\right)+\psi\left(b_{2}\right) \delta\left(b_{2}-x\right)$. By using the representation (18) we construct the map

$$
J^{b / a}: \mathscr{C} \rightarrow \Phi(X, \mathbf{C})
$$

The $J^{b / a}$ maps contexts (complexes of, e.g., physical conditions) into complex amplitudes. The representation (17) of probability as the square of the absolute value of the complex $(b / a)$-amplitude is nothing other than the famous Born rule.

Remark. We underline that the complex linear space representation (18) of the set of contexts $\mathscr{C}$ is based on a pair $(a, b)$ of incompatible (Kolmogorovian) random variables. Here $\psi_{C}=\psi_{C}^{b / a}$. We call random variables $a, b$ reference variables. Such a pair of variables determines a "probabilistic system of coordinates" on a contextual Kolmogorov space.

The complex amplitude $\psi_{C}(x)$ can be called a wave function of the complex of physical conditions, context $C$ or a (pure) quantum state. In principle, we can represent each context $C \in \mathscr{C}$ by a family of complex amplitudes:

$$
\psi(x) \equiv \psi_{C}(x)=\sum_{y \in Y} \sqrt{p_{C}^{a}(y) p(x / y)} e^{i \xi_{C}(x / y)}
$$

such that $\xi_{C}\left(x / a_{1}\right)-\xi_{C}\left(x / a_{2}\right)=\theta_{C}(x)$. For such complex amplitudes we also have Born's rule (17). However, to simplify considerations we shall consider only the representation (18) and the map (19) induced by this representation.

\section{Hilbert space representation of the $b$-variable}

We set $e_{x}^{b}(\cdot)=\delta(x-\cdot)$. For any context $C \in \mathscr{C}$, the complex amplitude $\psi_{C}$ can be expanded as:

$$
\psi_{C}=\sum_{x \in X} \psi_{C}(x) e_{x}^{b}
$$

Thus the Born's rule for complex amplitudes (17) can be rewritten in the following form:

$$
p_{C}^{b}(x)=\left|\left(\psi_{C}, e_{x}^{b}\right)\right|^{2},
$$

where the scalar product in the space $E=\Phi(X, C)$ is defined by the standard formula:

$$
(\psi, \psi)=\sum_{x \in X} \psi(x) \bar{\psi}(x)
$$

The system of functions $\left\{e_{x}^{b}\right\}_{x \in X}$ is an orthonormal basis in the Hilbert space $H=(E,(\cdot, \cdot))$.

\footnotetext{
15 To fix a representation of a contextual Kolmogorov space $\mathscr{K}$ we should fix phases. We shall see that to obtain a "good representation" we should choose phases in a special way.
} 
Let $X \subset \mathbf{R}$. By using the Hilbert space representation (22) of the Born's rule we obtain the Hilbert space representation of the expectation of the (Kolmogorovian) random variable $b$ :

$$
E(b / C)=\sum_{x \in X} x p_{C}^{b}(x)=\sum_{x \in X} x\left|\psi_{C}(x)\right|^{2}=\sum_{x \in X} x\left(\psi_{C}, e_{x}^{b}\right) \overline{\left(\psi_{C}, e_{x}^{b}\right)}=\left(\hat{b} \psi_{C}, \psi_{C}\right),
$$

where the (self-adjoint) operator $\hat{b}: H \rightarrow H$ is determined by its eigenvectors: $\hat{b}_{x}^{b}=x e_{x}^{b}, x \in X$. This is the multiplication operator in the space of complex functions $\Phi(X, \mathbf{C})$ :

$$
\hat{b} \psi(x)=x \psi(x)
$$

By (24) the conditional expectation of the Kolmogorovian random variable $b$ is represented with the aid of the selfadjoint operator $\hat{b}$. Therefore it is natural to represent this random variable (in the Hilbert space model) by the operator $\hat{b}$. So the Hilbert space image $\hat{b}$ of the random variable $b$ was defined through the formula (24) for conditional average. This formula is a simple consequence of the Born's rule (22). And the Born's rule is present in a latent form in the formula of total probability with interference term (13). This formula induces the representation of a context $C$ by the complex amplitude $\psi_{C}$ defined by (18). The amplitude has a natural expansion with respect to the basis $\left\{e_{x}^{b}\right\}_{x \in X}$, see (21). And this basis induces the representation (24).

We would like to introduce an operator $\hat{a}$ representing the random variable $a$ by using similar arguments. But we emphasize that random variables $a$ and $b$ do not play the same role in the Hilbert space representation under consideration. In fact, we now consider the $b / a$-projection of $\mathscr{K}$.

\section{Born's rule for the $a$-variable}

We start with the complex amplitude $\psi_{C}$ defined by (18). We shall see that this amplitude can be expanded with respect to a natural basis, $\left\{e_{y}^{a}\right\}_{y \in Y}$. That expansion plays the role similar to the expansion (21) with respect to the basis $\left\{e_{x}^{b}\right\}_{x \in X}$, namely Born's rule takes place for the $a$-variable (under a natural restriction to the matrix of transition probabilities):

$$
p_{C}^{a}(y)=\left|\left(\psi, e_{y}^{a}\right)\right|^{2}, y \in Y .
$$

We set:

$$
u_{j}^{a}=\sqrt{p_{C}^{a}\left(a_{j}\right)}, u_{j}^{b}=\sqrt{p_{C}^{b}\left(b_{j}\right)}, p_{i j}=p\left(b_{j} / a_{i}\right), u_{i j}=\sqrt{p_{i j}}, \theta_{j}=\theta_{C}\left(b_{j}\right), e_{j}^{b}=e_{b_{j}}^{b}, e_{j}^{a}=e_{a_{j}}^{a} .
$$

We remark that the coefficients $u_{j}^{a}, u_{j}^{b}$ depend on a context $C$; so $u_{j}^{a}=u_{j}^{a}(C), u_{j}^{b}=u_{j}^{b}(C)$. We also consider the matrix of transition probabilities $\mathbf{P}^{b / a}=\left(p_{i j}\right)$. It is always a stochastic matrix. ${ }^{16}$ We have, see (21), that

$$
\psi_{C}=v_{1}^{b} e_{1}^{b}+v_{2}^{b} e_{2}^{b} \text {, where } v_{j}^{b}=u_{1}^{a} u_{1 j}+u_{2}^{a} u_{2 j} e^{i \theta_{j}} .
$$

Hence

$$
p_{C}^{b}\left(b_{j}\right)=\left|v_{j}^{b}\right|^{2}=\left|u_{1}^{a} u_{1 j}+u_{2}^{a} u_{2 j} e^{i \theta_{j}}\right|^{2} .
$$

This is the interference representation of probabilities that is used, e.g., in quantum formalism. ${ }^{17}$

For any context $C_{0}$, we can represent the corresponding wave function $\psi=\psi_{C_{0}}$ in the form:

$$
\psi=u_{1}^{a} e_{1}^{a}+u_{2}^{a} e_{2}^{a}
$$

where

$$
e_{1}^{a}=\left(u_{11}, u_{12}\right), e_{2}^{a}=\left(e^{i \theta_{1}} u_{21}, e^{i \theta_{2}} u_{22}\right)
$$

We suppose that vectors $\left\{e_{i}^{a}\right\}$ are linearly independent, so $\left\{e_{i}^{a}\right\}$ is a basis in $H$. We have:

$$
e_{1}^{a}=v_{11} e_{1}^{b}+v_{12} e_{2}^{b}, \quad e_{2}^{a}=v_{21} e_{1}^{b}+v_{22} e_{2}^{b}
$$

\footnotetext{
${ }^{16}$ So $p_{i 1}+p_{i 2}=1, i=1,2$.

${ }^{17}$ By starting with the general representation (20) we obtain $v_{j}^{b}=u_{1}^{a} u_{1 j} e^{i \xi_{1 j}}+u_{2}^{a} u_{2 j} e^{i \xi_{2 j}}$ and the interference representation $p_{C}^{b}\left(b_{j}\right)=\left|v_{j}^{b}\right|^{2}=$ $\mid u_{1}^{a} u_{1 j} e^{i \xi_{1 j}}+u_{2}^{a} u_{2 j} e^{\left.i \xi_{2 j}\right|^{2}}$.
} 
Here $V=\left(v_{i j}\right)$ is the matrix: $v_{11}=u_{11}, v_{21}=u_{21}$ and $v_{12}=e^{i \theta_{1}} u_{21}, v_{22}=e^{i \theta_{2}} u_{22}$. We would like to find a class of matrixes $V$ such that Born's rule (25) holds. By (28) we have the Born's rule (25) iff $\left\{e_{i}^{a}\right\}$ was an orthonormal basis, i.e., the $V$ was a unitary matrix. Since we study the two-dimensional case (i.e., dichotomous random variables), $V \equiv V^{b / a}$ is unitary iff the matrix of transition probabilities $\mathbf{P}^{b / a}$ is double stochastic and $e^{i \theta_{1}}=-e^{i \theta_{2}}$ or

$$
\theta_{C_{0}}\left(b_{1}\right)-\theta_{C_{0}}\left(b_{2}\right)=\pi \bmod 2 \pi
$$

We recall that a matrix is double stochastic if it is stochastic, i.e., $p_{j 1}+p_{j 2}=1$, and, moreover,

$$
p_{1 j}+p_{2 j}=1, j=1,2 \text {. }
$$

Double stochasticity is equivalent to the condition: $p_{11}=p_{22}, p_{12}=p_{21}$. Any matrix of transition probabilities is stochastic (as a consequence of additivity of the conditional probability), but in general it is not double stochastic. We remark that the constraint (30) on phases and the double stochasticity constraint (31) are not independent:

Lemma 2. Let $a$ and $b$ be incompatible random variables and let the matrix of transition probabilities $\mathbf{P}^{b / a}$ be double stochastic. Then:

$$
\cos \theta_{C}\left(b_{2}\right)=-\cos \theta_{C}\left(b_{1}\right)
$$

for any context $C \in \mathscr{C}$.

Proof. By Lemma 1 we have:

$$
\sum_{x \in X} \cos \theta_{C}(x) \sqrt{\Pi_{y \in Y} p_{C}^{a}(y) p(x / y)}=0
$$

But for a double stochastic matrix $\mathbf{P}^{b / a}=(p(x / y))$ we have:

$$
\Pi_{y \in Y} p_{C}^{a}\left(a_{1}\right) p\left(b_{1} / y\right)=\Pi_{y \in Y} p_{C}^{a}\left(a_{2}\right) p\left(b_{2} / y\right) .
$$

Since random variables $a$ and $b$ are incompatible, we have $p(x / y) \neq 0, x \in X, y \in Y$. Since $C \in \mathscr{C}_{a}$, we have $p_{C}^{a}(y) \neq$ $0, y \in Y$. We obtain (32).

By Lemma 2 we have two different possibilities to choose phases:

$$
\theta_{C_{0}}\left(b_{1}\right)+\theta_{C_{0}}\left(b_{2}\right)=\pi \text { or } \theta_{\mathrm{C}_{0}}\left(\mathrm{~b}_{1}\right)-\theta_{\mathrm{C}_{0}}\left(\mathrm{~b}_{2}\right)=\pi \bmod 2 \pi
$$

By (30) to obtain the Born's rule for the $a$-variable we should choose phases $\theta_{C_{0}}\left(b_{i}\right), i=1,2$, in such a way that

$$
\theta_{C_{0}}\left(b_{2}\right)=\theta_{C_{0}}\left(b_{1}\right)+\pi
$$

If $\theta_{C_{0}}\left(b_{1}\right) \in[0, \pi]$ then $\theta_{C_{0}}\left(b_{2}\right) \in[\pi, 2 \pi]$ and vice versa. Lemma 2 is very important since by it (in the case when reference observables are chosen in such way that the matrix of transition probabilities is double stochastic) we can always choose $\theta_{C_{0}}\left(b_{j}\right), j=1,2$, to satisfy (33).

The delicate feature of the presented construction of the $a$-representation is that the basis $e_{y}^{a}$ depends on the context $C_{0}: e_{y}^{a}=e_{y}^{a}\left(C_{0}\right)$. And the Born's rule, in fact, has the form:

$$
p_{C_{0}}^{a}(y)=\left|\left(\psi_{C_{0}}, e_{y}^{a}\left(C_{0}\right)\right)\right|^{2}
$$

We would like to use (as in the conventional quantum formalism) one fixed $a$-basis for all contexts $C \in \mathscr{C}$. We may try to use for all contexts $C \in \mathscr{C}$ the basis $e_{y}^{a} \equiv e_{y}^{a}\left(C_{0}\right)$ corresponding to one fixed context $C_{0}$. We shall see that this is really the fruitful strategy.

Lemma 3. Let $\mathbf{P}^{b / a}$ be double stochastic and let for any context $C \in \mathscr{C}$ phases $\theta_{C}\left(b_{j}\right)$ be chosen as

$$
\theta_{C}\left(b_{2}\right)=\theta_{C}\left(b_{1}\right)+\pi \bmod 2 \pi .
$$

Then for any context $C \in \mathscr{C}$ we have the Born's rule (25) for the basis $e_{y}^{a} \equiv e_{y}^{a}\left(C_{0}\right)$ constructed for a fixed context $C_{0}$.

Proof. Let $C_{0} \in \mathscr{C}$. We take the basic $\left\{e_{y}^{a}\left(C_{0}\right)\right\}$ (and the matric $V\left(C_{0}\right)$ ) corresponding to this context. For any $C \in \mathscr{C}$, we would like to represent the wave function $\psi_{C}$ as

$$
\psi_{C}=v_{1}^{a}(C) e_{1}^{a}\left(C_{0}\right)+v_{2}^{a}(C) e_{2}^{a}\left(C_{0}\right), \text { where }\left|v_{j}^{a}(C)\right|^{2}=p_{C}^{a}\left(a_{j}\right)
$$


It is clear that, for any $C \in \mathscr{C}$, we can represent the wave function as

$$
\begin{aligned}
& \psi_{C}\left(b_{1}\right)=u_{1}^{a}(C) v_{11}\left(C_{0}\right)+e^{i\left[\theta_{C}\left(b_{1}\right)-\theta_{C_{0}}\left(b_{1}\right)\right]} u_{2}^{a}(C) v_{12}\left(C_{0}\right) \\
& \psi_{C}\left(b_{2}\right)=u_{1}^{a}(C) v_{21}\left(C_{0}\right)+e^{i\left[\theta_{C}\left(b_{2}\right)-\theta_{C_{0}}\left(b_{2}\right)\right]} u_{2}^{a}(C) v_{22}\left(C_{0}\right)
\end{aligned}
$$

Thus to obtain (35) we should have:

$$
\theta_{C}\left(b_{1}\right)-\theta_{C_{0}}\left(b_{1}\right)=\theta_{C}\left(b_{2}\right)-\theta_{C_{0}}\left(b_{2}\right) \bmod 2 \pi
$$

for any pair of contexts $C_{0}$ and $C_{1}$. By using the relations (34) between phases $\theta_{C}\left(b_{1}\right), \theta_{C}\left(b_{2}\right)$ and $\theta_{C_{0}}\left(b_{1}\right), \theta_{C_{0}}\left(b_{2}\right)$ we obtain:

$$
\theta_{C}\left(b_{2}\right)-\theta_{C_{0}}\left(b_{2}\right)=\left(\theta_{C}\left(b_{1}\right)+\pi-\theta_{C_{0}}\left(b_{1}\right)-\pi\right)=\theta_{C}\left(b_{1}\right)-\theta_{C_{0}}\left(b_{1}\right) \quad \bmod 2 \pi
$$

The constraint (34) essentially restricted the class of complex amplitudes which can be used to represent a context $C \in \mathscr{C}$. Any $C$ can be represented only by two amplitudes $\psi(x)$ and $\bar{\psi}(x)$ corresponding to the two possible choices of $\theta_{C}\left(b_{1}\right):$ in $[0, \pi]$ or $(\pi, 2 \pi)$.

By Lemma 3 we obtain the following result playing the fundamental role in our approach:

Theorem 2. We can construct the complex Hilbert space representation of the contextual Kolmogorov probability model such that the Born's rule holds for both reference variables iff the matrix of transition probabilities $\mathbf{P}^{b / a}$ is double stochastic.

If $\mathbf{P}^{b / a}$ is double stochastic we have the quantum representation not only for the classical conditional expectation of the variable $b$, see (24), but also for the variable $a$ :

$$
E(a / C)=\sum_{y \in Y} y p_{C}^{a}(y)=\sum_{y \in Y} y\left|\left(\psi_{C}, e_{y}^{a}\right)\right|^{2}=\left(\hat{a} \psi_{C}, \psi_{C}\right)
$$

where the self-adjoint operator (symmetric matrix) $\hat{a}: H \rightarrow H$ is determined by its eigenvectors: $\hat{a} e_{y}^{a}=y e_{y}^{a}$. By (37) it is natural to represent the random variable $a$ by the operator $\hat{a}$. Of course, the representation of random variables by linear operators is just a convenient mathematical tool to represent the average of a random variable by using only the Hilbert space structure.

Let us denote the unit sphere in the Hilbert space $H$ by the symbol $S$. The map $J^{b / a}: \mathscr{C} \rightarrow S$ need not be a surjection (injection). In general the set of (pure) states corresponding to a contextual Kolmogorov space $S_{\mathscr{C}} \equiv S_{\mathscr{C}}^{b / a}=J^{b / a}(\mathscr{C})$ is just a proper subset of the sphere $S$. The structure of the set of pure states $S_{\mathscr{C}}$ is determined by the Kolmogorov space.

\section{Some properties of the quantum projection}

Let $\mathbf{P}^{b / a}$ be double stochastic and let phases be chosen according to (34).

The contexts $A_{y}$ are degenerate with respect to the $a$-variable, since $\mathbf{P}\left(A_{a_{1}} \cap A_{a_{2}}\right)=0$. Thus $J^{b / a}\left(A_{i}\right)$ cannot be defined by (18). It is natural to extend the map $J^{b / a}$ to sets $A_{y}$ by setting $J^{b / a}\left(A_{y}\right)=e_{y}^{a}, y \in Y$. We set

$$
\overline{\mathscr{C}}=\mathscr{C} \cup \mathscr{A}, \mathscr{A}=\left\{A_{a_{1}}, A_{a_{2}}\right\}
$$

Thus we have constructed the Hilbert space representation: $J^{b / a}: \overline{\mathscr{C}} \rightarrow S$. We set $S_{\overline{\mathscr{C}}}=J^{b / a \overline{\mathscr{C}}}$.

Let $\delta(x / a, C)=0, i=1,2 .{ }^{18}$ Here $\lambda(x / a, C)=0$ and hence (for $\left.x \in X\right): \theta_{C}\left(b_{1}\right)=\frac{\pi}{2}$ or $\theta_{C}\left(b_{1}\right)=\frac{3}{2} \pi$. In the first case we have

$$
\begin{aligned}
& \psi_{C}\left(b_{1}\right)=\sqrt{p_{C}^{a}\left(a_{1}\right) p\left(b_{1} / a_{1}\right)}+i \sqrt{p_{C}^{a}\left(a_{2}\right) p\left(b_{1} / a_{2}\right)} \\
& \psi_{C}\left(b_{2}\right)=\sqrt{p_{C}^{a}\left(a_{1}\right) p\left(b_{2} / a_{1}\right)}-i \sqrt{p_{C}^{a}\left(a_{2}\right) p\left(b_{2} / a_{2}\right)}
\end{aligned}
$$

\footnotetext{
18 We remark that by Lemma 1 the sum of perturbation coefficients $\delta(x / a, C)$ is always equal to zero. Thus those coefficients are equal to zero or distinct from zero at the same time.
} 
The second choice of phases gives the representation of $C$ by the complex amplitude which is conjugate to (38). We set

$$
\mathscr{C}_{0}=\{C \in \mathscr{C}: \delta(x / a, C)=0, x \in X\} .
$$

We remark that $\Omega$ always belong to $\mathscr{C}_{0}$. However, in general $\mathscr{C}_{0} \neq\{\Omega\}$. By considering contexts $C \in \mathscr{C}_{0}$ we would not find any sign of the latent quantum structure in the classical probability space. But it should be underlined that $\mathscr{C}_{0} \equiv \mathscr{C}_{0}(a, b)$. Thus there can exist another pair of incompatible rrandom variables, $a^{\prime}, b^{\prime}$ such that they produce nontrivial interference for a context $C \in \mathscr{C}_{0}(a, b)$.

Let $C_{1}, C_{2} \in \mathscr{C}$ be contexts such that probability distributions of random variables $a$ and $b$ under $C_{1}$ and $C_{2}$, respectively, coincide:

$$
p_{C_{1}}^{a}(y)=p_{C_{2}}^{a}(y), y \in Y, p_{C_{1}}^{b}(x)=p_{C_{2}}^{b}(x), x \in X .
$$

In such a case $\lambda\left(x / a, C_{1}\right)=\lambda\left(x / a, C_{2}\right)$ and $\theta\left(x / a, C_{1}\right)= \pm \theta\left(x / a, C_{2}\right)$. If there is such a coincidence of probability distributions for only a pair of contexts $\left(C_{1}, C_{2}\right)$, then we can represent $C_{1}$ and $C_{2}$ by two different complex amplitudes, $\psi_{C_{2}}=\bar{\psi}_{C_{1}}$. But if we have the coincidence for a triple of contexts $\left(C_{1}, C_{2}, C_{3}\right)$ then it would be impossible to represent them by different complex amplitudes. We should choose $\psi_{C_{3}}=\psi_{C_{1}}$ or $\psi_{C_{3}}=\psi_{C_{2}}$; so $J^{b / a}\left(C_{3}\right)=J^{b / a}\left(C_{1}\right)$ or $J^{b / a}\left(C_{3}\right)=J^{b / a}\left(C_{2}\right)$. Thus in general the map $J^{b / a}$ is not injective.

\section{NONQUANTUM HILBERT SPACE PROJECTIONS OF THE CONTEXTUAL KOLMOGOROV MODEL}

Of course, for arbitrary random variables $a$ and $b$ the matrix $\mathbf{P}^{b / a}$ need not be double stochastic. In this case we could not obtain Born's rule both for the $b$ and $a$ variables. In general, for each random variable we should introduce its own scalar product and corresponding Hilbert space: $H_{b}=\left(E,(\cdot, \cdot)_{b}\right), H_{a}=\left(E,(\cdot, \cdot)_{a}\right), \ldots$, where $(\psi, \phi)_{b}=$ $\sum_{j} v_{j}^{b} \bar{w}_{j}^{b}$ for $\psi=\sum_{j} v_{j}^{b} e_{j}^{b}, \phi=\sum_{j} w_{j} e_{j}^{b}$, and $(\psi, \phi)_{a}=\sum_{j} v_{j}^{a} \bar{w}_{j}^{a}$ for $\psi=\sum_{j} v_{j}^{a} e_{j}^{a}, \phi=\sum_{j} w_{j}^{a} e_{j}^{a}$. The Hilbert spaces $H_{b}, H_{a}, \ldots$ give the $b$-representation, the $a$-representation, .... Thus $p_{C}^{b}(x)=\left|\left(\psi, e_{x}^{b}\right)_{b}\right|^{2}$ and $p_{C}^{a}(y)=\left|\left(\psi, e_{y}^{a}\right)_{a}\right|^{2}$ and so on.

However, the cruicial difficulty is that, as we have already discussed, $e_{y}^{a}=e_{y}^{a}\left(C_{0}\right)$ and, in fact, for any context $C_{0} \in \mathscr{C}$ we constructed its own Hilbert space representation for the $a$-variable: $H_{a}=H_{a}\left(C_{0}\right)$. In the same way as in the above considerations we would be able to use the same representation for contexts $C$ and $C_{0}$ if the condition (36) holds true. Thus we should have:

$$
\theta_{C}\left(b_{2}\right)=\theta_{C}\left(b_{1}\right)+\alpha \text { and } \theta_{\mathrm{C}_{0}}\left(\mathrm{~b}_{2}\right)=\theta_{\mathrm{C}_{0}}\left(\mathrm{~b}_{1}\right)+\alpha \bmod 2 \pi,
$$

where $\alpha$ is some phase (if $\mathbf{P}^{b / a}$ is double stochastic then $\alpha=\pi$ ).

Theorem 3. Suppose that $\mathbf{P}^{b / a}$ is not double stochastic and $\mathscr{C} \neq \mathscr{C}_{0}$. Then there is no such an $\alpha$ that

$$
\theta_{C}\left(b_{2}\right)=\theta_{C}\left(b_{1}\right)+\alpha
$$

for all contexts $C \in \mathscr{C}$.

To prove this theorem we need the following generalization of Lemma 2 :

Lemma 2a. Let $a$ and $b$ be incompatible random variables. Then for any context $C \in \mathscr{C}$ the following equality holds true:

$$
\cos \theta_{C}\left(b_{2}\right)=-k \cos \theta_{C}\left(b_{1}\right)
$$

where

$$
k \equiv k^{b / a}=\sqrt{\frac{p_{11} p_{21}}{p_{12} p_{22}}}
$$

It is also easy to obtain:

Proposition 1. The coefficient $k^{b / a}=1$ iff $\mathbf{P}^{b / a}$ is double stochastic.

Proof of Theorem. By Lemma 2a we have: $-k \cos \theta_{C}\left(b_{1}\right)=\cos \left(\theta_{C}\left(b_{1}\right)+\alpha\right)$ We take $C=\Omega$ and obtain: $\cos \left(\theta_{\Omega}\left(b_{1}\right)+\alpha\right)=0$. But $\theta_{\Omega}\left(b_{1}\right)= \pm \frac{\pi}{2}$. Thus $\theta_{\Omega}\left(b_{1}\right)+\alpha= \pm \frac{\pi}{2}$ and $\alpha=0, \pi \bmod 2 \pi$.

Since $\mathscr{C} \neq \mathscr{C}_{0}$ there exists a context $C$ such that $\cos \theta_{C}\left(b_{1}\right) \neq 0$. If $\alpha=0$ then $\cos \theta_{C}\left(b_{1}\right)(k+1)=0$. This contradicts to the positivity of $k$. Let $\alpha=\pi$. Then $\cos \theta_{C}\left(b_{1}\right)(k-1)=0$. Thus $k=1$. But this implies (by Proposition 1) that $\mathbf{P}^{b / a}$ is double stochastic. 
Despite Theorem 3, we can still hope that there can be found some extended family $\mathscr{C}^{\prime}$ of contexts such that (39) would hold true for contexts $C \in \mathscr{C}^{\prime}$. But it is impossible:

Proposition 2. Let condition (39) hold true for two contexts $C_{1}, C_{2}$ such that

$$
\left|\lambda\left(b_{1} / a, C_{1}\right)\right| \neq\left|\lambda\left(b_{2} / a, C_{2}\right)\right| .
$$

Then $\mathbf{P}^{b / a}$ is double stochastic.

Proof. We set $\theta=\theta_{C_{1}}\left(b_{1}\right)$ and $\theta^{\prime}=\theta_{C_{2}}\left(b_{1}\right)$. We have: $-k \cos \theta=\cos (\theta+\alpha),-k \cos \theta^{\prime}=\cos \left(\theta^{\prime}+\alpha\right)$. Thus

$$
-k \cos \frac{\theta+\theta^{\prime}}{2} \cos \frac{\theta-\theta^{\prime}}{2}=\cos \left(\frac{\theta+\theta^{\prime}}{2}+\alpha\right) \cos \frac{\theta-\theta^{\prime}}{2} .
$$

By (41) we have that $\cos \frac{\theta-\theta^{\prime}}{2} \neq 0$ and hence $-k \cos \frac{\theta+\theta^{\prime}}{2}=\cos \left(\frac{\theta+\theta^{\prime}}{2}+\alpha\right)$.

We also have

$$
k \sin \frac{\theta+\theta^{\prime}}{2} \sin \frac{\theta-\theta^{\prime}}{2}=-\sin \left(\frac{\theta+\theta^{\prime}}{2}+\alpha\right) \sin \frac{\theta-\theta^{\prime}}{2} .
$$

By (41) we have that $\sin \frac{\theta-\theta^{\prime}}{2} \neq 0$ and hence $-k \sin \frac{\theta+\theta^{\prime}}{2}=\sin \left(\frac{\theta+\theta^{\prime}}{2}+\alpha\right)$. Thus $k^{2}=1$ and hence $k=1$. By proposition 1 the matrix $\mathbf{P}^{b / a}$ is double stochastic.

Thus if $\mathbf{P}^{b / a}$ is not double stochastic then every surface $M_{t}=\left\{C \in \mathscr{C}:\left|\lambda\left(b_{1} / a, C\right)\right|=t\right\}, 0 \leq t \leq 1$, in the space of contexts is represented in its own Hilbert space $H_{a}(t)$.

\section{NONCOMMUTATIVITY OF OPERATORS REPRESENTING KOLMOGOROVIAN RANDOM VARIABLES}

Let $\mathbf{P}^{b / a}$ be double stochastic and let phases be chosen according to (34). We consider in this section the case of real valued random variables. Here spectra of random variables $b$ and $a$ are subsets of $\mathbf{R}$. We set $q_{1}=\sqrt{p_{11}}=\sqrt{p_{22}}$ and $q_{2}=\sqrt{p_{12}}=\sqrt{p_{21}}$. Thus the vectors of the $a$-basis, see (29), have the following form:

$$
e_{1}^{a}=\left(q_{1}, q_{2}\right), e_{2}^{a}=\left(e^{i \theta_{1}} q_{2}, e^{i \theta_{2}} q_{1}\right) .
$$

Since $\theta_{2}=\theta_{1}+\pi$, we get $e_{2}^{a}=e^{i \theta_{2}}\left(-q_{2}, q_{1}\right)$. We now find matrices of operators $\hat{a}$ and $\hat{b}$ in the $b$-representation. The latter one is diagonal. For $\hat{a}$ we have: $\hat{a}=V \operatorname{diag}\left(\mathrm{a}_{1}, \mathrm{a}_{2}\right) \mathrm{V}^{\star}$, where $v_{11}=v_{22}=q_{1}, v_{21}=-v_{12}=q_{2}$. Thus $a_{11}=a_{1} q_{1}^{2}+a_{2} q_{2}^{2}, a_{22}=a_{1} q_{2}^{2}+a_{2} q_{1}^{2}, a_{12}=a_{21}=\left(a_{1}-a_{2}\right) q_{1} q_{2}$. Hence

$$
[\hat{b}, \hat{a}]=\hat{m},
$$

where $m_{11}=m_{22}=0$ and $m_{12}=-m_{21}=\left(a_{1}-a_{2}\right)\left(b_{2}-b_{1}\right) q_{1} q_{2}$. Since $a_{1} \neq a_{2}, b_{1} \neq b_{2}$ and $q_{j} \neq 0$, we have $\hat{m} \neq 0$.

\section{THE ROLE OF SIMULTANEOUS DOUBLE STOCHASTICITY OF $\mathbf{P}^{B / A}$ AND $\mathbf{P}^{A / B}$}

Starting with the $b$-representation - complex amplitudes $\psi_{C}(x)$ defined on the spectrum (range of values) of a random variable $b$-we constructed the $a$-representation. This construction is natural (i.e., it produces the Born's probability rule) only if the $\mathbf{P}^{b / a}$ is double stochastic. We would like to have a symmetric model. So by starting with the $a$ representation - complex amplitudes $\psi_{C}(y)$ defined on the spectrum (range of values) of a random variable $a$ - we would like to construct the natural $b$-representation. Thus both matrices of transition probabilities $\mathbf{P}^{b / a}$ and $\mathbf{P}^{a / b}$ should be double stochastic.

We set $B_{j}=B_{b_{j}}, A_{j}=A_{a_{j}}, j=1,2$. It is assumed that phases are always chosen according to (34).

Theorem 4. Let the matrix $\mathbf{P}^{b / a}$ be double stochastic. The contexts $B_{1}, B_{2}$ belong to $\mathscr{C}$ iff the matrix $\mathbf{P}^{a / b}$ is double stochastic.

Proof. We have

$$
\lambda\left(B_{2} / a, B_{1}\right)=-\frac{\mu_{1}^{2}+\mu_{2}^{2}}{2 \mu_{1} \mu_{2}}
$$


where $\mu_{j}=\sqrt{p_{B_{1}}^{a}\left(a_{j}\right) p\left(b_{2} / a_{j}\right)}$. So $\lambda\left(B_{2} / a, B_{1}\right) \geq 1$ and we have the trigonometric behavior only in the case $\mu_{1}=\mu_{2}$. Thus: $p_{B_{1}}^{a}\left(a_{1}\right) p\left(b_{2} / a_{1}\right)=p_{B_{1}}^{a}\left(a_{2}\right) p\left(b_{2} / a_{2}\right)$. In this case $\lambda\left(B_{2} / a, B_{1}\right)=-1$, so we can choose, e.g. $\theta\left(B_{2} / a, B_{1}\right)=\pi$, and consequently $\theta\left(B_{1} / a, B_{1}\right)=0$. We pay attention to the fact that $p_{B_{i}}^{a}\left(a_{j}\right)=p^{a / b}\left(a_{j} / b_{i}\right) \equiv p\left(a_{j} / b_{i}\right)$. Thus we have:

$$
p\left(a_{1} / b_{1}\right) p\left(b_{2} / a_{1}\right)=p\left(a_{2} / b_{1}\right) p\left(b_{2} / a_{2}\right) .
$$

In the same way by using conditioning with respect to $B_{2}$ we obtain: $p\left(a_{1} / b_{2}\right) p\left(b_{1} / a_{1}\right)=p\left(a_{2} / b_{2}\right) p\left(b_{1} / a_{2}\right)$. By using double stochasticity of $\mathbf{P}^{b / a}$ we can rewrite the last equality as

$$
p\left(a_{1} / b_{2}\right) p\left(b_{2} / a_{2}\right)=p\left(a_{2} / b_{2}\right) p\left(b_{2} / a_{1}\right) .
$$

Thus by (42) and (43) we have:

$$
\frac{p\left(a_{1} / b_{2}\right)}{p\left(a_{2} / b_{1}\right)}=\frac{p\left(a_{2} / b_{2}\right)}{p\left(a_{1} / b_{1}\right)}
$$

Hence $p\left(a_{1} / b_{2}\right)=t p\left(a_{2} / b_{1}\right)$ and $p\left(a_{2} / b_{2}\right)=t p\left(a_{1} / b_{1}\right), t>0$. But $1=p\left(a_{1} / b_{2}\right)+p\left(a_{2} / b_{2}\right)=t\left[p\left(a_{2} / b_{1}\right)+\right.$ $\left.p\left(a_{1} / b_{1}\right)\right]=t$.

To finish the proof, we need the following well known result:

Lemma 4. Both matrices of transition probabilities $\mathbf{P}^{b / a}$ and $\mathbf{P}^{a / b}$ are double stochastic iff the transition probabilities are symmetric, i.e.,

$$
p\left(b_{i} / a_{j}\right)=p\left(a_{j} / b_{i}\right), i, j=1,2 .
$$

This is equivalent that random variables $a$ and $b$ have the uniform probability distribution:

$$
p^{a}\left(a_{i}\right)=p^{b}\left(b_{i}\right)=1 / 2, i=1,2 .
$$

This Lemma has important physical consequences. A natural (Bornian) Hilbert space representation of contexts can be constructed only on the basis of a pair of (incompatible) uniformly distributed random variables.

Lemma 5. Let both matrices $\mathbf{P}^{b / a}$ and $\mathbf{P}^{a / b}$ be double stochastic. Then

$$
\lambda\left(B_{i} / a, B_{i}\right)=1 .
$$

Proof. Here $\delta\left(B_{i} / a, B_{i}\right)=1-p\left(b_{i} / a_{1}\right) p\left(a_{1} / b_{i}\right)-p\left(b_{i} / a_{2}\right) p\left(a_{2} / b_{i}\right)=1-p\left(a_{1} / b_{i}\right)^{2}-p\left(a_{2} / b_{i}\right)^{2}=2 p\left(a_{1} / b_{i}\right) p\left(a_{2} / b_{i}\right)$. Thus $\lambda\left(B_{i} / a, B_{i}\right)=1$. By (45) we have

$$
\lambda\left(B_{i} / a, B_{j}\right)=-1, i \neq j
$$

Thus

$$
\theta\left(B_{i} / a, B_{i}\right)=0 \text { and } \theta\left(B_{i} / a, B_{j}\right)=\pi, i \neq j .
$$

Proposition 3. Let $\mathbf{P}^{b / a}$ and $\mathbf{P}^{a / b}$ be double stochastic. Then

$$
J^{b / a}\left(B_{j}\right)(x)=\delta\left(b_{j}-x\right), x \in X, \text { and } J^{a / b}\left(A_{j}\right)(y)=\delta\left(a_{j}-y\right), y \in Y .
$$

Proof. Because $\theta\left(B_{1} / a, B_{1}\right)=0$ we have:

$$
\begin{gathered}
J^{b / a}\left(B_{1}\right)\left(b_{1}\right)=\sqrt{p\left(a_{1} / b_{1}\right) p\left(b_{1} / a_{1}\right)}+e^{i 0} \sqrt{p\left(a_{2} / b_{1}\right) p\left(b_{1} / a_{2}\right)} \\
=p\left(a_{1} / b_{1}\right)+p\left(a_{2} / b_{1}\right)=1 .
\end{gathered}
$$

Because $\theta\left(B_{2} / a, B_{1}\right)=\pi$ we have

$$
\begin{gathered}
J^{b / a}\left(B_{1}\right)\left(b_{2}\right)=\sqrt{p\left(a_{1} / b_{1}\right) p\left(b_{2} / a_{1}\right)}+e^{i \pi} \sqrt{p\left(a_{2} / b_{1}\right) p\left(b_{2} / a_{2}\right)} \\
=\sqrt{p\left(a_{1} / b_{1}\right)}\left(\sqrt{p\left(b_{2} / a_{1}\right.}-\sqrt{p\left(a_{2} / b_{1}\right)}\right)=0 .
\end{gathered}
$$

Thus in this case: $J^{b / a}\left(B_{i}\right)=e_{i}^{b}, i=1,2$.

Thus in the case when both matrices of transition probabilities $\mathbf{P}^{a / b}$ and $\mathbf{P}^{b / a}$ are double stochastic (i.e., both reference variables $a$ and $b$ are uniformly distributed) the Born's rule has the form:

$$
p_{C}^{b}(x)=\left|\left(\psi_{C}, \psi_{B_{x}}\right)\right|^{2}, p_{C}^{a}(y)=\left|\left(\psi_{C}, \psi_{A_{y}}\right)\right|^{2} .
$$

In principle, we could start directly with constructing a quantum-like representation of the constextual Kolmogorovian model in the case of uniformly distributed reference variables. In this case the whole construction looks more natural. But we started with the general representation based on an arbitrary pair of incompatible reference variables to see how general the formalism can be, cf. Mackey [14]. 


\section{EXAMPLE}

We consider an example of a Kolmogorov probability space and a pair of dichotomous random variables $a, b$ which are incompatible. In this example the set of contexts with nontrivial disturbance term $\delta, \delta \neq 0$, is nonempty, so $\mathscr{C}_{0} \neq \mathscr{C}$. We find the image $S_{\mathscr{C}}$ of the set of contexts $\mathscr{C}$ in the Hilbert sphere $S \subset H$. In this example $S_{\mathscr{C}}$ is a proper subset of the sphere $S$. The Hilbert space representation map $J^{b / a}$ is not injective. Random variables $a$ and $b$ are represented by symmetric operators in the Hilbert space $H$. They do not commute.

Let $\Omega=\left\{\omega_{1}, \omega_{2}, \omega_{3}, \omega_{4}\right\}$ and $\mathbf{P}\left(\omega_{j}\right)=p_{j}>0, \sum_{j=1}^{4} p_{j}=1$. Let

$$
\begin{aligned}
& A_{1}=\left\{\omega_{1}, \omega_{2}\right\}, A_{2}=\left\{\omega_{3}, \omega_{4}\right\} \\
& B_{1}=\left\{\omega_{1}, \omega_{4}\right\}, B_{2}=\left\{\omega_{2}, \omega_{3}\right\}
\end{aligned}
$$

Let $p_{1}=p_{3}=q<\frac{1}{2}$ and $p_{2}=p_{4}=(1-2 q) / 2$. We denote this Kolmogorov probability space by the symbol $\mathscr{K}(q)$.

Here $\mathbf{P}\left(A_{1}\right)=\mathbf{P}\left(A_{2}\right)=\mathbf{P}\left(B_{1}\right)=\mathbf{P}\left(B_{2}\right)=\frac{1}{2}$. So the random variables $a$ and $b$ are uniformly distributed. Thus both matrices of transition probabilities $\mathbf{P}^{b / a}$ and $\mathbf{P}^{a / b}$ are double stochastic. Here

$$
\mathbf{P}^{b / a}=\mathbf{P}^{a / b}=\left(\begin{array}{lr}
2 q & 1-2 q \\
1-2 q & 2 q
\end{array}\right)
$$

We have the symmetry condition $\mathbf{P}\left(B_{i} / A_{j}\right)=\mathbf{P}\left(A_{j} / B_{i}\right)$.

We start with two-points contexts.

(a) Let $C=C_{24}=\left\{\omega_{2}, \omega_{4}\right\}$. Here $\mathbf{P}(C)=1-2 q, \mathbf{P}\left(B_{j} / C\right)=\mathbf{P}\left(A_{j} / C\right)=\frac{1}{2}$. Thus $\delta=0$. By using the representation (38) and choosing $\theta_{C_{24}}\left(b_{1}\right)=\frac{\pi}{2}, \theta_{C_{24}}\left(b_{2}\right)=\frac{3 \pi}{2}$ we obtain:

$$
\psi_{C_{24}}(x)=\left\{\begin{array}{l}
\sqrt{q}+i \sqrt{\frac{1-2 q}{2}}, x=b_{1} \\
\sqrt{\frac{1-2 q}{2}}-i \sqrt{q}, x=b_{2}
\end{array}\right.
$$

(b). Let $C=C_{13}=\left\{\omega_{1}, \omega_{3}\right\}$. Here everything is as in (a). We set $\theta_{C_{13}}\left(b_{1}\right)=\frac{3}{2} \pi$ and $\theta_{C_{13}}\left(b_{2}\right)=\frac{\pi}{2}$. Thus

$$
\psi_{C_{13}}(x)=\left\{\begin{array}{l}
\sqrt{q}-i \sqrt{\frac{1-2 q}{2}}, x=b_{1} \\
\sqrt{\frac{1-2 q}{2}}+i \sqrt{q}, x=b_{2}
\end{array}\right.
$$

We remark that $\psi_{C_{24}} \perp \psi_{C_{13}}$ :

$$
\left(\psi_{C_{24}}, \psi_{C_{13}}\right)=\left(\sqrt{q}+i \sqrt{\frac{1-2 q}{2}}\right)^{2}+\left(\sqrt{\frac{1-2 q}{2}}-i \sqrt{q}\right)^{2}=0
$$

(c) Let $C=C_{14}=\left\{\omega_{1}, \omega_{4}\right\}=B_{1}$. By general theory we have $\psi_{C_{14}}(x)=\delta\left(b_{1}-x\right)=e_{1}^{b}$. In the same way: $\psi_{C_{23}}=\delta\left(b_{2}-x\right)=e_{2}^{b}$.

To find the Hilbert space representation of sets $C=C_{12}=\left\{\omega_{1}, \omega_{2}\right\}=A_{1}$ and $C=C_{34}=\left\{\omega_{3}, \omega_{4}\right\}=A_{2}$ we have to construct the basis $\left\{e_{j}^{a}\right\}$. We can choose:

$$
e_{1}^{a}=\left(\begin{array}{c}
\sqrt{2 q} \\
\sqrt{1-2 q}
\end{array}\right) e_{2}^{a}=i\left(\begin{array}{c}
-\sqrt{1-2 q} \\
\sqrt{2 q}
\end{array}\right)
$$

Here we have chosen $\theta_{2}=\theta_{C_{13}}\left(b_{2}\right)=\frac{\pi}{2}$. Thus we shall use the $a$-basis corresponding to the context $C_{0} \equiv C_{13}$. We have $\psi_{C_{12}}=e_{1}^{a}, \psi_{C_{34}}=e_{2}^{a}$. In a) and b) we found the probabilistic amplitudes representing contexts $C_{24}$ and $C_{13}$ in the $b$-basis. In the $a$-basis those amplitudes are represented by

$$
\psi_{C_{24}}=\frac{1}{\sqrt{2}}\left[e_{1}^{a}-e_{2}^{a}\right], \psi_{C_{13}}=\frac{1}{\sqrt{2}}\left[e_{1}^{a}+e_{2}^{a}\right]
$$


(d) Let $C=C_{123}=\left\{\omega_{1}, \omega_{2}, \omega_{3}\right\}$. Here $\mathbf{P}(C)=(2 q+1) / 2, \mathbf{P}\left(A_{1} / C\right)=\mathbf{P}\left(B_{2} / C\right)=1 /(2 q+1), \mathbf{P}\left(A_{2} / C\right)=$ $\mathbf{P}\left(B_{1} / C\right)=2 q /(2 q+1)$. Thus $\delta\left(B_{1} / a, C\right)=\frac{2 q(2 q-1)}{2 q+1}$ and, hence, $\lambda\left(B_{1} / a, C\right)=-\frac{\sqrt{1-2 q}}{2}$. This context is trigonometric: $C_{123} \in \mathscr{C}$. We remark that $\lambda\left(B_{2} / a, C\right)=\frac{\sqrt{1-2 q}}{2}$ (since $\mathbf{P}^{b / a}$ is double stochastic). ${ }^{19}$ We choose $\theta_{2}=\arccos \frac{\sqrt{1-2 q}}{2}$, so $\theta_{1}=\arccos \frac{\sqrt{1-2 q}}{2}-\pi$. We have:

$$
\psi_{C_{123}}(x)=\left\{\begin{array}{l}
\sqrt{\frac{2 q}{2 q+1}}-e^{i \arccos \frac{\sqrt{1-2 q}}{2}} \sqrt{\frac{2 q(1-2 q)}{2 q+1}}, x=b_{1} \\
\sqrt{\frac{1-2 q}{2 q+1}}+e^{i \arccos \frac{\sqrt{1-2 q}}{2}} \frac{2 q}{\sqrt{2 q+1}}, \quad x=b_{2}
\end{array}\right.
$$

Thus

$$
\psi_{C_{123}}=\frac{1}{\sqrt{2 q+1}} e_{1}^{a}-i e^{i \arccos \frac{\sqrt{1-2 q}}{2}} \sqrt{\frac{2 q}{2 q+1}} e_{2}^{a} .
$$

(e) Let $C=C_{124}=\left\{\omega_{1}, \omega_{2}, \omega_{4}\right\}$. Here $\mathbf{P}(C)=1-q, \mathbf{P}\left(A_{1} / C\right)=\mathbf{P}\left(B_{1} / C\right)=1 / 2(1-q), \mathbf{P}\left(A_{2} / C\right)=\mathbf{P}\left(B_{2} / C\right)=$ $(1-2 q) / 2(1-q)$. Thus $\delta\left(B_{1} / a, C\right)=q(1-2 q) /(1-q)$ and, hence, $\lambda\left(B_{1} / a, C\right)=\sqrt{\frac{q}{2}}<1$, and the context $C_{124} \in \mathscr{C}$. We choose $\theta_{1}=\arccos \sqrt{\frac{1}{2}}$, so $\theta_{2}=\arccos \sqrt{\frac{1}{2}}+\pi$. Thus:

$$
\begin{aligned}
& \psi_{C_{124}}(x)=\left\{\begin{array}{l}
\sqrt{\frac{q}{1-q}}+e^{i \arccos \sqrt{\frac{q}{2}}} \frac{1-2 q}{\sqrt{2(1-q)}}, \quad x=b_{1} \\
\sqrt{\frac{1-2 q}{2(1-q)}}-e^{i \arccos \sqrt{\frac{q}{2}} \sqrt{\frac{q(1-2 q)}{1-q}}, x=b_{2}}
\end{array}\right. \\
& \psi_{C_{124}}(x)=\frac{1}{\sqrt{2(1-q)}} e_{1}^{a}+i e^{i \arccos \sqrt{\frac{q}{2}}} \sqrt{\frac{1-2 q}{2(1-q)}} e_{2}^{a} .
\end{aligned}
$$

(f) Let $C=C_{234}=\left\{\omega_{2}, \omega_{3}, \omega_{4}\right\}$. Here $\mathbf{P}(C)=1-q, \mathbf{P}\left(A_{1} / C\right)=\mathbf{P}\left(B_{1} / C\right)=(1-2 q) / 2(1-q), \mathbf{P}\left(A_{2} / C\right)=$ $\mathbf{P}\left(B_{2} / C\right)=1 / 2(1-q)$. Thus $\delta\left(B_{1} / a, C\right)=q(2 q-1) /(1-q)$ and, hence, $\lambda\left(B_{1} / a, C\right)=-\sqrt{\frac{q}{2}}, \lambda\left(B_{2} / a, C\right)=\sqrt{\frac{q}{2}}$. Here:

$$
\begin{aligned}
& \psi_{C_{234}}(x)=\left\{\begin{array}{l}
\sqrt{\frac{q(1-2 q)}{1-q}}-e^{i \arccos \sqrt{\frac{q}{2}}} \sqrt{\frac{1-2 q}{2(1-q)}}, x=b_{1} \\
\frac{1-2 q}{\sqrt{2(1-q)}}+e^{i \arccos \sqrt{\frac{q}{2}}} \sqrt{\frac{q}{1-q}}, \quad x=b_{2}
\end{array}\right. \\
& \psi_{C_{234}}(x)=\sqrt{\frac{1-2 q}{2(1-q)}} e_{1}^{a}-i e^{i \arccos \sqrt{\frac{q}{2}}} \frac{1}{\sqrt{2(1-q)}} e_{2}^{a} .
\end{aligned}
$$

(g) Let $C=C_{134}=\left\{\omega_{1}, \omega_{3}, \omega_{4}\right\}$. Here $\mathbf{P}(C)=(2 q+1) / 2, \mathbf{P}\left(A_{1} / C\right)=\mathbf{P}\left(B_{2} / C\right)=2 q /(2 q+1), \mathbf{P}\left(A_{2} / C\right)=$ $\mathbf{P}\left(B_{1} / C\right)=1 /(2 q+1)$. Thus $\delta\left(B_{1} / a, C\right)=2 q(1-2 q) /(2 q+1)$ and, hence, $\lambda\left(B_{1} / a, C\right)=\frac{\sqrt{1-2 q}}{2}$. Thus:

$$
\begin{gathered}
\psi_{C_{134}}(x)=\left\{\begin{array}{l}
\frac{2 q}{\sqrt{2 q+1}}+e^{i \arccos \frac{\sqrt{1-2 q}}{2}} \sqrt{\frac{1-2 q}{2 q+1}}, \quad x=b_{1} \\
\sqrt{\frac{2 q(1-2 q)}{2 q+1}}-e^{i \arccos \frac{\sqrt{1-2 q}}{2}} \sqrt{\frac{2 q}{2 q+1}}, x=b_{2}
\end{array}\right. \\
\psi_{C_{134}}=\sqrt{\frac{2 q}{2 q+1}} e_{1}^{a}+e^{i \arccos \frac{\sqrt{1-q}}{2}} \frac{1}{\sqrt{2 q+1}} e_{2}^{a} .
\end{gathered}
$$

(h) Let $C=\Omega$. Here we know from the beginning that $\delta\left(B_{j} / a, C\right)=0$. Here $\mathbf{P}\left(A_{i} / C\right)=\mathbf{P}\left(A_{i}\right)=1 / 2$ and $\mathbf{P}\left(B_{i} / C\right)=$ $\mathbf{P}\left(B_{i}\right)=1 / 2$. We can choose the phase $\theta_{\Omega}\left(b_{1}\right)=\frac{\pi}{2}$ or $\frac{3 \pi}{2}$. The first choice of gives the complex amplitude $\psi_{\Omega}=\psi_{C_{24}}$ and the second $\psi_{\Omega}=\psi_{C_{13}}$. Thus for both representations the map $J^{b / a}$ is not injective.

19 We pay attention on the dependence of $\theta=\arccos \frac{\sqrt{1-2 q}}{2}$ on the parameter $q: \theta(q)$ increases from $\pi / 3$ to $\pi / 2$, when $q$ increases from 0 to $1 / 2$. 
The representation map $J^{b / a}$ transforms the conventional probabilistic calculus in $\mathscr{K}$ to the quantum probabilistic calculus in $H$. For example, let a random variable $b= \pm 1$. Here

$$
E\left(b / C_{234}\right)=\mathbf{P}\left(B_{1} / C_{234}\right)-\mathbf{P}\left(B_{2} / C_{234}\right)=\frac{1-2 q}{2(1-q)}-\frac{1}{2(1-q)}=\frac{q}{q-1} .
$$

Hence the conventional probabilistic calculus gives the answer $q /(q-1)$. But we also have:

$$
\begin{gathered}
<\hat{b}>_{\psi_{C_{234}}}=\left(\hat{b} \psi_{C_{234}}, \psi_{C_{234}}\right) \\
=\left|\sqrt{\frac{q(1-2 q)}{1-q}}-e^{i \arccos \sqrt{\frac{q}{2}}} \sqrt{\frac{1-2 q}{2(1-q)}}\right|^{2} \\
-\left|\sqrt{\frac{(1-2 q)^{2}}{2(1-q)}}+e^{i \arccos \sqrt{\frac{q}{2}}} \sqrt{\frac{q}{1-q}}\right|^{2} \\
=\frac{q(1-2 q)}{1-q}+\frac{1-2 q}{2(1-q)}-2 \sqrt{\frac{q}{2} \frac{q(1-2 q)}{1-q}} \frac{1-2 q}{2(1-q)} \\
-\frac{(1-2 q)^{2}}{2(1-q)}-\frac{q}{1-q}-2 \sqrt{\frac{q}{2} \frac{(1-2 q)^{2}}{2(1-q)} \frac{q}{1-q}}=\frac{q}{q-1} .
\end{gathered}
$$

Thus the quantum probabilistic calculus gives us the same result $q /(q-1)$. In the same way we have for a random variable $a= \pm 1$ :

$$
\begin{gathered}
E\left(a / C_{234}\right)=\mathbf{P}\left(A_{1} / C_{234}\right)-\mathbf{P}\left(A_{2} / C_{234}\right)=\frac{q}{q-1} \\
<\hat{a}>_{\psi_{C_{234}}}=\left(\hat{a} \psi_{C_{234}}, \psi_{C_{234}}\right)=\left|\sqrt{\frac{1-2 q}{2(1-q)}}\right|^{2}-\left|-i e^{i \arccos \sqrt{\frac{q}{2}}} \frac{1}{\sqrt{2(1-q)}}\right|^{2}=\frac{q}{q-1} .
\end{gathered}
$$

In this example the set of nonsensitive contexts contains three contexts: $\mathscr{C}_{0}=\left\{\Omega, C_{24}, C_{13}\right\}$. We have

$$
S_{\bar{C}}=\left\{\psi_{C_{13}}, \psi_{C_{24}}, \psi_{C_{14}}=e_{1}^{b}, \psi_{C_{23}}=e_{2}^{b}, \psi_{C_{12}}=e_{1}^{a}, \psi_{C_{23}}=e_{2}^{a}, \psi_{C_{124}}, \psi_{C_{234}}, \psi_{C_{123}}, \psi_{C_{134}}\right\}
$$

Therefore the set of pure states $S_{\overline{\mathscr{C}}}$ is a finite, ten-points, subset of the unit sphere in the two dimensional Hilbert space. We remark that all vectors in $S_{\bar{C}}$ are pairwise independent.

There is the parameter $q \in(0,1 / 2)$ determining a Kolmogorov probability space $\mathscr{K}(q)$. For each value of $q$ we have a finite set of pure states. However, a family $\mathscr{K}(q), q \in(0,1 / 2)$, of Kolmogorov probability spaces generates a "continuous" set $\cup_{q} S_{\overline{\mathscr{C}}}(q)$ of pure states.

Finally, we remark that we have chosen one fixed representation of every Kolmogorov model $\mathscr{K}(q)$ (for fixed $q)$. We can construct other representations corresponding to other choices of phases. For example, we can construct another representation by choosing $\theta_{C_{24}}\left(b_{1}\right)=\frac{3 \pi}{2}, \theta_{C_{13}}\left(b_{1}\right)=\frac{\pi}{2}, \theta_{C_{134}}\left(b_{1}\right)=-\arccos \frac{\sqrt{1-2 q}}{2}$,

$\theta_{C_{123}}\left(b_{2}\right)=-\arccos \frac{\sqrt{1-2 q}}{2}, \theta_{C_{124}}\left(b_{1}\right)=-\arccos \sqrt{\frac{q}{2}}, \theta_{C_{234}}\left(b_{2}\right)=-\arccos \sqrt{\frac{q}{2}}$, as well as combine some phases choices of various representations.

\section{HILBERT SPACE IMAGES OF THE REFERENCE RANDOM VARIABLES}

We begin with the following standard definition:

Definition 3. For a self-adjoint operator $\hat{d}$ the quantum mean value in the state $\psi$ is defined by

$$
\langle\hat{d}\rangle_{\psi}=(\hat{d} \psi, \psi) .
$$

Theorem 5. For any map $f: \mathbf{R} \rightarrow \mathbf{R}$, we have:

$$
\langle f(\hat{a})\rangle_{\psi_{C}}=E(f(a) / C),\langle f(\hat{b})\rangle_{\psi_{C}}=E(f(b) / C)
$$


for any context $C \in \overline{\mathscr{C}}$.

Proof. By using the Born's rule for the $b$ we obtain:

$$
E\left(f(b / C)=\sum_{x \in X} f(x) p_{c}^{b}(x)=\sum_{x \in X} f(x)\left|\left(\psi_{C}, e_{x}^{b}\right)\right|^{2}=\langle f(\hat{b})\rangle_{\psi_{C}}\right.
$$

The same result we have for the $f(\hat{a})$ since (as $\mathbf{P}^{b / a}$ is double stochastic) we have Born's probability rule both for $b$ and $a$.

Theorem 6. Let $f, g: \mathbf{R} \rightarrow \mathbf{R}$ be two arbitrary functions. Then

$$
E(f(a)+g(b) / C)=\langle f(\hat{a})+g(\hat{b})\rangle_{\psi_{C}}
$$

for any context $C \in \overline{\mathscr{C}}$.

Proof. By using linearity of the Kolmogorov mathematical expectation, Theorem 3, and linearity of the Hilbert space scalar product we obtain:

$$
\begin{gathered}
E(f(a(\omega))+g(b(\omega)) / C)=E(f(a(\omega) / C)+E(g(b(\omega)) / C) \\
=\langle f(\hat{a})\rangle_{\psi_{C}}+\langle g(\hat{b})\rangle_{\psi_{C}}=\langle f(\hat{a})+g(\hat{b})\rangle_{\psi_{C}}
\end{gathered}
$$

Denote the linear space of all random variables of the form $d(\omega)=f(a(\omega))+g(b(\omega))$ by the symbol $\mathscr{O}_{+}(a, b)$ and the linear space of operators of the form $\hat{d}=f(\hat{a})+g(\hat{b})$ by $\mathscr{O}_{+}(\hat{a}, \hat{b})$.

Theorem 7. The map $T=T^{a / b}: \mathscr{O}_{+}(a, b) \rightarrow \mathscr{O}_{+}(\hat{a}, \hat{b}), d=f(a)+g(b) \rightarrow \hat{d}=f(\hat{a})+g(\hat{b})$, preserves the conditional expectation:

$$
\langle T(d)\rangle_{\psi_{C}}=(T(d) J(C), J(C))=E(d / C) .
$$

The transformation $T$ preserves the conditional expectation for random variables $d \in \mathscr{O}_{+}(a, b)$. But in general we cannot expect anything more, since in general $T$ does not preserve probability distributions. The important problem is to extend the map $T$ for a larger class (linear space?) of Kolmogorovian random variables with preserving (48). It is natural to define (as we always do in the conventional quantum formalism):

$$
T(f)(\hat{a}, \hat{b})=f(\hat{a}, \hat{b})
$$

where $f(\hat{a}, \hat{b})$ is the pseudo differential operator with the Weyl symbol $f(a, b)$. We shall see that already for $f(a, b)=$ $a b$ (so $f(\hat{a}, \hat{b})=(\hat{a} \hat{b}+\hat{b} \hat{a}) / 2)$ the equality (48) is violated.

We can consider the $b$ and the $a$ as discrete analogues of the position and momentum observables. The operators $\hat{b}$ and $\hat{a}$ give the Hilbert space (quantum) representation of these observables. We also introduce an analogue of the energy observable:

$$
\mathscr{H}(\omega)=\frac{a^{2}(\omega)}{2 m}+V(b(\omega)),
$$

where $V: \mathbf{R} \rightarrow \mathbf{R}$ is a map. The Hilbert space representation of this observable is given by the operator of energy (Hamiltonian)

$$
\hat{\mathscr{H}}=\frac{\hat{a}^{2}}{2 m}+V(\hat{b}) .
$$

By Theorem 7 for contexts $C \in \overline{\mathscr{C}}$ the averages of the observables $\mathscr{H}(\omega)$ (Kolmogorovian) and $\hat{\mathscr{H}}$ (quantum) coincide:

$$
E(\mathscr{H}(\omega) / C)=\langle\mathscr{H}\rangle_{\psi_{C}} .
$$

However, as we shall see, probability distributions do not coincide:

Proposition 4. There exists context $C$ such that the probability distribution of the random variable $d(\omega)=a(\omega)+$ $b(\omega)$ with respect to $C$ does not coincide with the probability distribution of the quantum observable $\hat{d}=\hat{a}+\hat{b}$ with respect to the state $\psi_{C}$ corresponding to $C$.

Proof. It suffices to present an example of such a context $C$. Take the context $C=C_{234}$ from the Example. We consider the case: $a(\omega)= \pm \gamma, b(\omega)= \pm \gamma, \gamma>0$; so $d(\omega)=-2 \gamma, 0,2 \gamma$. Corresponding Kolmogorovian probabilities can easily be found:

$$
p_{C}^{d}(-2 \gamma)=q /(1-q), p_{C}^{d}(0)=(1-2 q) /(1-q), p_{C}^{d}(2 \gamma)=0 .
$$


We now find the probability distribution of $\hat{d}$. To do this, we find eigenvalues and eigenvectors of the self-adjoint operator $\hat{d}$. We find the matrix of the operator $\hat{d}$ in the basis $\left\{e_{j}^{b}\right\}: d_{11}=-d_{22}=4 q \gamma$ and $d_{12}=d_{21}=2 \gamma \sqrt{2 q(1-2 q)}$. We have $k_{1,2}= \pm 2 \sqrt{2 q} \gamma$. Of course, the range of values of the quantum observable $\hat{d}$ differs from the range of values of the random variable $d$. However, this difference of ranges of values is not so large problem in this case. The random variable $d$ takes only two values, $-2 \gamma, 0$ with the probability one. Moreover, we can represent values of the quantum observable $\hat{d}$ as just an affine transform of values of the random variable $d$ :

$$
d_{\text {quantum }}=2 \sqrt{2 q} d-\gamma .
$$

In principle we can interpret such a transformation as representing some special measurement procedure. Thus in this example the problem with spectrum is not crucial. The crucial problem is that $d$ and $\hat{d}$ have different probability distributions.

Corresponding eigenvectors are

$$
\begin{aligned}
& e_{1}^{d}=\frac{1}{\sqrt{2(1-\sqrt{2 q})}}(-\sqrt{1-2 q}, \sqrt{2 q}-1) \\
& e_{2}^{d}=\frac{1}{\sqrt{2(1+\sqrt{2 q})}}(-\sqrt{1-2 q}, \sqrt{2 q}+1)
\end{aligned}
$$

Finally, we find (by using the expression for $\psi_{C_{234}}$ which was found in section 6):

$$
\begin{aligned}
& p_{c}^{\hat{d}}\left(k_{1}\right)=\left|\left(\psi_{C}, e_{1}^{d}\right)\right|^{2}=\frac{(1-\sqrt{2 q})(2+\sqrt{2 q})}{4(1-q)} \\
& p_{c}^{\hat{d}}\left(k_{2}\right)=\left|\left(\psi_{C}, e_{2}^{d}\right)\right|^{2}=\frac{(1+\sqrt{2 q})(2-\sqrt{2 q})}{4(1-q)}
\end{aligned}
$$

Thus $d$ and $\hat{d}$ have essentially different probability distributions.

\section{DISPERSION-FREE STATES}

As originally stated by von Neumann, [5] the problem of hidden variables is to find whether dispersion free states exist in quantum mechanics. He answered the question in the negative. The problem of the existence of dispersion free states as well as von Neumann's solution were the subject of great debates. We do not want to go into detail see, e.g., [11]. In our contextual approach an analogue of this problem can be formulated as: Do dispersion free contexts exist?

The answer is the positive. In the Example we can take any atom of the Kolmogorov probability space $\mathscr{K}_{q}$, e.g., $C=\left\{\omega_{1}\right\}$. Since, for any random variable $\xi$ on the Kolmogorov space $\mathscr{K}_{q}$, it has a constant value on such a $C$ the dispersion of $\xi$ under the context $C$ is equal to zero:

$$
D(\xi / C)=E\left[(\xi-E(\xi / C))^{2} / C\right]=0 .
$$

However, dispersion free contexts do not belong to the system $\overline{\mathscr{C}}$ of contexts which can be mapped by $J^{a / b}$ into the Hilbert space $H$. On the one hand, our contextual approach gives the possibility to have the realist viewpoint to quantum mechanics.. On the other hand, it does not contradict to the von Neumann as well as other "no-go" theorems. The mathematical representation of contexts (complexes of physical conditions) given by the quantum formalism it too rough to represent dispersion free contexts.

Conclusion: Dispersion free contexts exist, but they could not be represented by quantum states (complex probabilistic amplitudes).

\section{CLASSICAL AND QUANTUM SPACES AS ROUGH IMAGES OF FUNDAMENTAL PRESPACE}

Our contextual probabilistic model induces the following picture of physical reality. 


\section{Prespace and classical space}

There exists a prespace $\Omega$ which points corresponds to primary (irreducible) states of physical systems, prestates or fundamental physical parameters. Functions $d: \Omega \rightarrow \mathbf{R}^{m}$ are said to be preobservables. The set of all preobservables is denoted by the symbol $\mathscr{O}_{p} \equiv \mathscr{O}_{p}(\Omega)$. We are not able (at least at the moment) to measure an arbitrary preobservable $d \in \mathscr{O}_{p}$.

Nevertheless, some preobservables can be measured. Suppose that there exists a preobservable $b$ such that all measurements can be reduced to some measurements of $b$, cf. L. De Broglie [12] and D. Bohm [8]. on the possibility to reduce any measurement to a position measurement. Let $X \subset \mathbf{R}^{m}$ be the range of values of $b$. The $X$ is said to be a classical space ${ }^{20}$. Set $B_{x}=\{\omega \in \Omega: b(\omega)=x\}=b^{-1}(x), x \in X$.

In principle a set $B_{x}$ could contain millions of points. Dynamics in $X$ is classical dynamics. In our model, classical dynamics is a rough image of dynamics in the prespace $\Omega^{21}$.

\section{Classical phase space}

Let $a$ be a preobservable which is incompatible with our fundamental preobservable $b$ (space observable). We denote by $Y \subset \mathbf{R}^{m}$ the range of values of the $a$. The $Y$ is said to be conjugate space to the classical space $X$. We call the $b$ position and the a momentum. We set $A_{y}=\{\omega \in \Omega: a(\omega)=y\}=a^{-1}(y), y \in Y$.

Since $A_{y}$ is not a subset of $B_{x}$ for any $x \in X$ (this is a consequence of incompatibility of the observables $a$ and $b$ ), the point $y$ cannot be used to get finer description of any point $x \in X$. Thus by using values of $a$ we cannot obtain a finer space structure. The variables $b$ and $a$ are really incompatible. By fixing the value of, e.g., $a=y_{0}$ we cannot fix the value of $b=x_{0}$.

Remark. (Nonlocal dependence of incompatible variables at the prespace level). Since, for a fixed $y_{0} \in Y$, we have $A_{y_{0}} \cap B_{x} \neq \emptyset$ for any $x \in X$, a value $y_{0}$ of the momentum can be determined only by all values $x \in X$ of the position. Thus on the level of the prespace incompatible variables are nonlocally dependent. However, this prespace nonlocality could not be found in classical mechanics, since in the latter the finer prespace structure is destroyed by the rough $(x, y)$ encoding.

The space $\Pi=X \times Y \subset \mathbf{R}^{2 m}$ is a classical phase space. Dynamics in the phase space gives a rough image in the terms of the two incompatible variables of dynamics in the prespace. The phase space $\Pi$ is a classical contextual $(b, a)$-picture of the prespace $\Omega$. This picture is richer than the pure $b$-space picture, $X$. The $\Pi$ contains images of the two families of contexts $\mathscr{A}=\left\{A_{y}\right\}$ and $\mathscr{B}=\left\{B_{x}\right\}$.

In our probabilistic investigations we have seen that the most natural choice of incompatible variables corresponds to random variables $a(\omega)$ and $b(\omega)$ which are uniformly distributed. On the other hand, the creation of a uniform partition of the prespace $\Omega$ is the most natural way to create a rough image $X$ of the prespace - a classical space.

As the next step we can consider statistical mechanics on the classical space $X$. In such a statistical theory from the very beginning we lost the finer statistical structure of the model based on probability distributions on the prespace. Functions $u: \Pi \rightarrow \mathbf{R}^{q}$ are called classical observables. The set of classical observables is denoted by the symbol $\mathscr{O}_{c}(\Pi)$. We shall also use symbols $\mathscr{O}_{c}(X)$ and $\mathscr{O}_{c}(Y)$ to denote spaces of classical observables depending only on the $b$-position and the $a$-momentum, respectively.

\section{Quantum mechanics and the Hilbert space representation of prespace contexts}

Neither classical nor quantum mechanics can describe the individual dynamics in the prespace. Of course, such a viewpoint to quantum mechanics contradicts to the so called orthodox Copenhagen interpretation by which the wave function describes an individual quantum system. It also contradicts to the conventional viewpoint to classical mechanics.

\footnotetext{
${ }^{20}$ Of course, in such a model the classical space $X$ depends on the preobservable $X \equiv X(b)$. Thus $X$ is the $b$-image of the prespace $\Omega$.

${ }^{21}$ Consider in the Example the trajectory $\omega_{1} \rightarrow \omega_{2} \rightarrow \omega_{3} \rightarrow \omega_{4} \rightarrow \omega_{1}$ in the $\Omega$. In the classical space $X$ this trajectory is represented by $b_{1} \rightarrow b_{1} \rightarrow b_{2} \rightarrow b_{2} \rightarrow b_{1}$.
} 
By our contextual interpretation the wave function has the realist prespace interpretation. A complex amplitude is nothing than the image (induced by the contextual formula of total probability) of a set of fundamental parameters - a context. Thus the Hilbert state space $H$ is not less real than the classical real space $\mathbf{R}^{3}$.

Observables which probability distributions can be found by using the representation by self-adjoint operators in the Hilbert space are called quantum observables. The set of quantum observables is denoted by the symbol $\mathscr{O}_{q}(H)$. Neither classical statistical nor quantum mechanics can provide knowledge about the probability distribution of an arbitrary preobservable. Nevertheless, the quantum theory gives some information about some preobservables, namely fundamental preobservable $b$ and $a$ and pre-observables $d$ belonging to the class $\mathscr{O}_{+}(a, b)$. Another way to look to the same problem is to say that the quantum theory (with our contextual probabilistic interpretation) gives the possibility to represent some prespace structures, namely some contexts $C \in \mathscr{C}$ by vectors of a Hilbert state space.

Neither classical nor quantum mechanics are fundamental theories. They could not give information about the point wise structure of the prespace $\Omega$. But the quantum formalism represents some complexes of physical conditions domains in the prespace - which are not represented in the classical space or phase space. Of course, the quantum formalism also represents classical position states $x \in X$ by wave functions $\psi_{B_{x}}$ (Hilbert states $e_{x}^{b}$ ). Classical states $x \in X$ are images of prespace contexts $B_{x}$. But the quantum formalism represents also some sets $C \subset \Omega$ which have no classical images (namely, images in $X$ or $\Pi$ ).

In the Example we take the set $C=C_{123}=\left\{\omega_{1}, \omega_{2}, \omega_{3}\right\}$. Neither $C \subset B_{1}$ nor $C \subset B_{2}$. This prespace domain $C$ can be described neither by the position $x=b_{1}$ nor $x=b_{2}$. The quantum state $\psi_{C} \in S \subset H$ representing this domain of the prespace describes the superposition of the two classical states $x=b_{1}$ and $x=b_{2}$. Hence a physical system prepared under the complex physical conditions $C=C_{123}$ is (from the classical viewpoint) in the superposition of two different positions.

\section{Heisenberg uncertainty principle}

We now take the context $C=A_{y}$ for some $y \in Y$. Here the momentum $a$ has the definite value. But $A_{y} \cap B_{x} \neq \emptyset$ for any $x \in X$. Hence the state $\psi_{C}=e_{y}^{a} \in H$ also corresponds to the superposition of two positions $x=b_{1}$ and $x=b_{2}$. This is nothing else than (the discrete analogue) the Heisenberg uncertainty principle. In the same way in any state with the definite position, $\psi_{C}=e_{x}^{b}, x \in X$, the momentum can not have the definite value.

Thus the Hilbert sphere $S$ contains images of the classical spaces $X$ and $Y$ (but not the phase space $\Pi$, see further considerations), $X \equiv\left\{e_{x}^{b}\right\} \subset S$ and $Y=\equiv\left\{e_{y}^{a}\right\} \subset S$. But the Hilbert space contains also images of nonclassical domains $C \in \overline{\mathscr{C}}$. We remark that (depending on the model) only a part of the Hilbert sphere corresponds to some domains of the prespace. All other quantum states, $\psi \notin S_{\overline{\mathscr{C}}}$, are just ideal mathematical objects which do no correspond to any context in the prespace.

As was already remarked, the phase space $\Pi$ is not imbedded into the Hilbert sphere $S$, since contexts $C_{x y}=B_{x} \cap A_{y}$ corresponding to points of the $\Pi$ do not belong to the system $\overline{\mathscr{C}}$ which is mapped into $S$ (because these contexts are degenerate with respect to both reference observables).

\section{Preobservables and quantum observables}

For what class of preobservables can we find probability distributions with respect to contexts $C \in \overline{\mathscr{C}}$ by using the quantum formalism? As we have seen, we are not able to find the probability distribution for an arbitrary preobservable $d \in \mathscr{O}_{p}(\Omega)$. In general the operators $\hat{d}=d(\hat{a}, \hat{b})$ corresponding to functions $d(x, y)$ (e.g., $d(x, y)=x y$ or $\left.d(x, y)=x+y\right)$ are not directly related to prequantum observables $d(\omega)=d(b(\omega), a(\omega))$.

Only quantum observables $\hat{d}=f(\hat{b})$ and $\hat{d}=g(\hat{a})$ have the same probability distributions as the corresponding preobservables $d(\omega)=f(b(\omega))$ and $d(\omega)=g(a(\omega))$. By Theorem 7 the average is preserved by the canonical map $T^{b / a}: \mathscr{O}_{+}(a, b) \rightarrow \mathscr{O}_{+}(\hat{a}, \hat{b})$.

However, even such quantum observables give just a rough image of corresponding preobservables. By using quantum probabilistic formalism we can find probability distributions only for quantum states $\psi_{C} \in S_{\overline{\mathscr{C}}} \subset H$. Those quantum states represent only some special contexts. Hence by using the quantum formalism we could not find the probability distribution of a preobservable $a(\omega)$ or $b(\omega)$ for an arbitrary context represented by a domain in the prespace $\Omega$. Neither we can reconstruct maps $a(\omega)$ and $b(\omega)$. Thus the quantum theory is not a fundamental 
theory. It does not provide the complete (even statistical) description of the prespace reality. However, some statistical information about the prespace structure can be obtained by using the quantum probabilistic formalism.

\section{On the mystery of operator quantization}

The origin of the operator quantization was always mysterious for me. Why the correspondence between functions and functions of operators (of the position and the momentum) provides the correct statistical description of quantum measurements? Our contextual model tells that the only reason is the coincidence of quantum averages with 'real' prespace (contextual) averages for some preobservables (in particular, of the form $f(b)+g(a)$ ).

Theorem 7 is only a sufficient condition for the coincidence of averages. But even such a result gives the possibility to connect the quantum Hamiltonian

$$
\hat{\mathscr{H}}=\frac{\hat{a}^{2}}{2 m}+V(\hat{b})
$$

with the realist preobservable $\mathscr{H}(a(\omega), b(\omega))=\frac{a(\omega)^{2}}{2 m}+V(b(\omega))$. Quantum averages of energy expressed by the Hilbert space averages of the Hamiltonian $\mathscr{\mathscr { H }}$ coincides with averages of the realist energy preobservable $\mathscr{H}(\omega)$. However, for some contexts $C$ quantum energy observable $\hat{\mathscr{H}}$ and energy preobservable $\mathscr{H}$ have different probability distributions, see Proposition 4. In principle, such an effect should be observable experimentally.

The classical space is a contextual image of the fundamental prespace $\Omega$. This is a very poor image since only a few special contexts namely space-contexts have images in the classical space $\mathbf{R}^{3}$. In principle, there might be created various classical spaces (corresponding to various fundamental variables) on the basis of the prespace $\Omega$. Human beings have been creating their own (very special) classical space. Since light rays play the fundamental role in the creating of our classical space it can be called electromagnetic classical space. So the electromagnetic classical space is created on the basis on electromagnetic reduction of information. In principle there can exist systems which are able to perform some other reductions of information, e.g., gravitation reduction. They would create a gravitational classical space.

\section{HYPERBOLIC HILBERT SPACE PROJECTION OF THE CLASSICAL PROBABILISTIC MODEL}

We study here the model with the hyperbolic interference. We set

$$
\mathscr{C}^{\text {hyp }}=\left\{C \in \mathscr{C}_{a}:\left|\lambda\left(B_{j} / a, c\right)\right| \geq 1, j=1,2\right\} .
$$

We call elements of $\mathscr{C}^{\text {hyp }}$ hyperbolic contexts.

\section{Hyperbolic algebra}

Instead of the field complex numbers $\mathbf{C}$, we shall use so called hyperbolic numbers, namely the two dimensional Clifford algebra, G, see [49]. We call this algebra hyperbolic algebra. ${ }^{22}$

Denote by the symbol $j$ the generator of the algebra $\mathbf{G}$ of hyperbolic numbers:

$$
j^{2}=1 .
$$

\footnotetext{
${ }^{22}$ Of course, it is rather dangerous to invent an own name for a notion established almost as firm as complex numbers. We use a new name, hyperbolic algebra, for the well known algebraic object, the two dimensional Clifford algebra, by following reasons. First we explain why we dislike to use the standard notion Clifford algebra in this particular case. The standard Clifford machinery was developed around noncommutative features of general Clifford algebras. The two dimensional Clifford algebra, hyperbolic algebra in our terminology, is commutative. Commutativity of $\mathbf{G}$ is very important in our considerations. We now explain why we propose the name hyperbolic algebra. Hyperbolic functions are naturally related to the algebraic structure of $\mathbf{G}$ through a hyperbolic generalization of Euler's formula for the complex numbers. This is the crucial point of our considerations - the possibility to use this algebraic structure to represent some special transformations for hyperbolic functions.
} 
The algebra $\mathbf{G}$ is the two dimensional real algebra with basis $e_{0}=1$ and $e_{1}=j$. Elements of $\mathbf{G}$ have the form $z=x+j y, x, y \in \mathbf{R}$. We have $z_{1}+z_{2}=\left(x_{1}+x_{2}\right)+j\left(y_{1}+y_{2}\right)$ and $z_{1} z_{2}=\left(x_{1} x_{2}+y_{1} y_{2}\right)+j\left(x_{1} y_{2}+x_{2} y_{1}\right)$. This algebra is commutative. It is not a field - not every element has the inverse one.

We introduce an involution in $\mathbf{G}$ by setting $\bar{z}=x-j y$ and set $|z|^{2}=z \bar{z}=x^{2}-y^{2}$. We remark that $|z|=\sqrt{x^{2}-y^{2}}$ is not well defined for an arbitrary $z \in \mathbf{G}$. We set $\mathbf{G}_{+}=\left\{z \in \mathbf{G}:|z|^{2} \geq 0\right\}$. We remark that $\mathbf{G}_{+}$is a multiplicative semigroup as follows from the equality

$\left|z_{1} z_{2}\right|^{2}=\left|z_{1}\right|^{2}\left|z_{2}\right|^{2}$.

Thus, for $z_{1}, z_{2} \in \mathbf{G}_{+}$, we have that $\left|z_{1} z_{2}\right|$ is well defined and $\left|z_{1} z_{2}\right|=\left|z_{1}\right|\left|z_{2}\right|$. We define a hyperbolic exponential function by using a hyperbolic analogue of the Euler's formula:

$e^{j \theta}=\cosh \theta+j \sinh \theta, \theta \in \mathbf{R}$.

We remark that

$e^{j \theta_{1}} e^{j \theta_{2}}=e^{j\left(\theta_{1}+\theta_{2}\right)}, \overline{e^{j \theta}}=e^{-j \theta},\left|e^{j \theta}\right|^{2}=\cosh ^{2} \theta-\sinh ^{2} \theta=1$.

Hence, $z= \pm e^{j \theta}$ always belongs to $\mathbf{G}_{+}$. We also have

$\cosh \theta=\frac{e^{j \theta}+e^{-j \theta}}{2}, \sinh \theta=\frac{e^{j \theta}-e^{-j \theta}}{2 j}$.

We set $\mathbf{G}_{+}^{*}=\left\{z \in \mathbf{G}_{+}:|z|^{2}>0\right\}$. Let $z \in \mathbf{G}_{+}^{*}$. We have

$z=|z|\left(\frac{x}{|z|}+j \frac{y}{|z|}\right)=\operatorname{sign} \mathrm{x}|\mathrm{z}|\left(\frac{\mathrm{xsignx}}{|\mathrm{z}|}+\mathrm{j} \frac{\mathrm{ysignx}}{|\mathrm{z}|}\right)$.

As $\frac{x^{2}}{|z|^{2}}-\frac{y^{2}}{|z|^{2}}=1$, we can represent $x \operatorname{sign} x=\cosh \theta$ and $y \operatorname{sign} x=\sinh \theta$, where the phase $\theta$ is unequally defined. We can represent each $z \in \mathbf{G}_{+}^{*}$ as

$z=\operatorname{sign} x|z| \mathrm{e}^{\mathrm{j} \theta}$.

By using this representation we can easily prove that $\mathbf{G}_{+}^{*}$ is a multiplicative group. Here $\frac{1}{z}=\frac{\operatorname{sign} x}{|z|} e^{-j \theta}$. The unit circle in $\mathbf{G}$ is defined as $S_{1}=\left\{z \in \mathbf{G}:|z|^{2}=1\right\}=\left\{z= \pm e^{j \theta}, \boldsymbol{\theta} \in(-\infty,+\infty)\right\}$. It is a multiplicative subgroup of $\mathbf{G}_{+}^{*}$.

\section{Hyperbolic probability amplitude, hyperbolic Born's rule}

The interference formula of total probability (15) can be written in the following form:

$$
p_{C}^{b}(x)=\sum_{y \in Y} p_{C}^{a}(y) p(x / y) \pm 2 \cosh \theta_{C}(x) \sqrt{\Pi_{y \in Y} p_{C}^{a}(y) p(x / y)}
$$

where $\theta_{C}(x)=\theta(x / a, C)= \pm \operatorname{arccosh}|\lambda(\mathrm{x} / \mathrm{a}, \mathrm{C})|, \mathrm{x} \in \mathrm{X}, \mathrm{C} \in \mathscr{C}^{\text {hyp }}$. Here the coefficient $\lambda$ is defined by (8). By using the elementary formula

$$
D=A+B \pm 2 A B \cosh \theta=\left|\sqrt{A} \pm e^{j \theta} \sqrt{B}\right|^{2},
$$

for $A, B>0$, we can represent the probability $p_{C}^{b}(x)$ as the square of the hyperbolic amplitude $p_{C}^{b}(x)=\left|\psi_{C}(x)\right|^{2}$, where

$$
\psi(x) \equiv \psi_{C}(x)=\sqrt{p_{C}^{a}\left(a_{1}\right) p\left(x / a_{1}\right)}+\varepsilon_{C}(x) e^{j \theta_{C}(x)} \sqrt{p_{C}^{a}\left(a_{2}\right) p\left(x / a_{2}\right)} .
$$

Here $\varepsilon_{C}(x)=\operatorname{sign} \delta(x / a, C)$. We remark that by Lemma 1 :

$$
\sum_{x \in X} \varepsilon_{C}(x)=0
$$

Thus we have a hyperbolic generalization of Born's rule for the $b$-variable.

\section{Hyperbolic Hilbert space representation}

Definition 4. A hyperbolic Hilbert space is $\mathbf{G}$-linear space (module) $H$ with a $\mathbf{G}$-linear scalar product: a map $(\cdot, \cdot): H \times H \rightarrow \mathbf{G}$ that is

1) linear with respect to the first argument:

$(a z+b w, u)=a(z, u)+b(w, u), a, b \in \mathbf{G}, z, w, u \in H ;$

2) symmetric: $(z, u)=\overline{(u, z)}$; 
3) nondegenerate: $(z, u)=0$ for all $u \in H$ iff $z=0$.

Remark. If we consider $H$ as just a $\mathbf{R}$-linear space, then $(\cdot, \cdot)$ is a bilinear form which is not positive defined. In particular, in the two dimensional case we have the signature: $(+,-,+,-)$.

We introduce on the space $\Phi(X, \mathbf{G})$ of functions: $\psi: X \rightarrow \mathbf{G}$. Since $X=\left\{b_{1}, b_{2}\right\}$, the $\Phi(X, \mathbf{G})$ is the two dimensional G-module. We define the $\mathbf{G}$-scalar product by (23) with conjugation in $\mathbf{G}$. The system of functions $\left\{e_{x}^{b}\right\}_{x \in X}$ is an orthonormal basis in the hyperbolic Hilbert space $H^{\text {hyp }}=(\Phi(X, \mathbf{G}),(\cdot, \cdot))$. Thus we have the hyperbolic Born's rule in $H^{\text {hyp }}$, see (22) - but with the hyperbolic scalar product. The random variable $b$ is represented by the multiplication operator $\hat{b}$ in $\Phi(X, \mathbf{G})$. We have the hyperbolic Hilbert space representation (24) of the average of $b$.

Thus we constructed a $\mathbf{G}$-linear representation of the contextual Kolmogorov model:

$$
J^{b / a}: \mathscr{C}^{\text {hyp }} \rightarrow H^{\text {hyp }}
$$

We set $S_{\mathscr{C} \text { hyp }}=J^{b / a}\left(\mathscr{C}^{\text {hyp }}\right)$. This is a subset of the unit sphere $S$ of the Hilbert space $H^{\text {hyp }}$.

By introducing the coefficients (26) and $\varepsilon_{i}=\varepsilon\left(b_{i}\right)$ we represent a state $\psi_{C}$ by $\psi_{C}=v_{1}^{b} e_{1}^{b}+v_{2}^{b} e_{2}^{b}$, where $v_{i}^{b}=$ $u_{1}^{a} u_{1 i}+\varepsilon_{i} u_{2}^{a} u_{2 i} e^{j \theta_{i}}$. So

$$
p_{C}^{b}\left(b_{i}\right)=\left|v_{i}^{b}\right|^{2}=\left|u_{1}^{a} u_{1 i}+\varepsilon_{i} u_{2}^{a} u_{2 i} e^{j \theta_{i}}\right|^{2} .
$$

This is the G-linear representation of the hyperbolic interference of probabilities. This formula can also be derived in the formalism of the hyperbolic Hilbert space. We remark that here the $\mathbf{G}$-linear combination $u_{1}^{a} u_{1 i}+\varepsilon_{i} u_{2}^{a} u_{2 i} e^{j \theta_{i}}$ belongs to $\mathbf{G}_{+}^{*}$.

Thus for any context $C_{0} \in \mathscr{C}^{\text {hyp }}$ we can represent $\psi_{C_{0}}$ in the form:

$$
\psi_{C_{0}}=u_{1}^{a} e_{1}^{a}+u_{2}^{a} e_{2}^{a}
$$

where

$$
e_{1}^{a}=\left(u_{11}, u_{12}\right), e_{2}^{a}=\left(\varepsilon_{1} e^{j \theta_{1}} u_{21}, \varepsilon_{2} e^{j \theta_{2}} u_{22}\right) .
$$

As in the $\mathbf{C}$-case, we introduce the matrix $V$ with coefficients $v_{11}=u_{11}, v_{21}=u_{21}$ and $v_{12}=\varepsilon_{1} e^{j \theta_{1}} u_{21}, v_{22}=\varepsilon_{2} e^{j \theta_{2}} u_{22}$. We remark that here coefficients $v_{i j} \in \mathbf{G}_{+}^{*}$. In the same way as in the complex case the Born's rule

$$
p_{C_{0}}^{a}\left(a_{i}\right)=\left|\left(\psi_{C_{0}}, e_{i}^{a}\right)\right|^{2}
$$

holds true in the $a$-basis iff $\left\{e_{i}^{a}\right\}$ is an orthonormal basis in $H^{\text {hyp }}$. The latter is equivalent to the G-unitary of the matrix $V$ (corresponding to the transition from $\left\{e_{i}^{b}\right\}$ to $\left.\left\{e_{i}^{a}\right\}\right): \bar{V}^{*} V=I$, or

$$
\begin{gathered}
\bar{v}_{11} v_{11}+\bar{v}_{21} v_{21}=1, \bar{v}_{12} v_{12}+\bar{v}_{22} v_{22}=1, \\
\bar{v}_{11} v_{12}+\bar{v}_{21} v_{22}=0 .
\end{gathered}
$$

Thus $1=u_{11}^{2}+u_{21}^{2}=p\left(b_{1} / a_{1}\right)+p\left(b_{1} / a_{2}\right)$ and $1=u_{12}^{2}+u_{22}^{2}=p\left(b_{2} / a_{1}\right)+p\left(b_{2} / a_{2}\right)$. Thus the first two equations of the $\mathbf{G}$-unitary are equivalent to the double stochasticity of $\mathbf{P}^{b / a}$ (as in the $\mathbf{C}$-case). We remark that the equations (53) can be written as

$$
\left|v_{11}\right|^{2}+\left|v_{21}\right|^{2}=1,\left|v_{12}\right|^{2}+\left|v_{22}\right|^{2}=1 \text {. }
$$

The third unitarity equation (54) can be written as

$$
u_{11} u_{12} \varepsilon_{1} e^{-j \theta_{2}}+u_{21} \varepsilon_{2} e^{-j \theta_{2}} u_{22}=0 .
$$

By using double stochasticity of $\mathbf{P}^{a / b}$ we obtain $e^{j \theta_{1}}=e^{j \theta_{2}}$. Thus

$$
\theta_{1}=\theta_{2}
$$

This is the hyperbolic analogue of the $\mathbf{C}$-unitary condition (30).

Lemma 6. Let $a$ and $b$ be incompatible random variables and let $\mathbf{P}^{b / a}$ be double stochastic. Then

$$
\cosh \theta_{C}\left(b_{2}\right)=\cosh \theta_{C}\left(b_{1}\right)
$$

for any context $C \in \mathscr{C}^{\text {hyp }}$. 
Proof. By Lemma 1 we have:

$$
\sum_{x} \varepsilon(x) \cosh \theta_{C}(x) \sqrt{\Pi_{y} p_{C}^{a}(y) p(x / y)}=0 .
$$

Double stochasticity of $\mathbf{P}^{b / a}$ implies (58).

The constraint (58) induced by double stochasticity can be written as the constraint to phases:

$$
\theta_{C}\left(b_{2}\right)= \pm \theta_{C}\left(b_{1}\right) .
$$

To obtain unitary of the matrix $V$ of transition $\left\{e_{i}^{b}\right\} \rightarrow\left\{e_{i}^{a}\right\}$ we should choose phases according to (57). And by (59) we can always do this for a double stochastic matrix of transition probabilities.

By choosing such a representation we obtain the hyperbolic generalization of the Born's rule (25) for the $a$-variable.

We now investigate the possibility to use one fixed basis $\left\{e_{j}^{a} \equiv e_{j}^{a}\left(C_{0}\right)\right\}, C_{0} \in \mathscr{C}^{\text {hyp }}$, for all states $\psi_{C}, C \in \mathscr{C}^{\text {hyp }}$. For any $C \in \mathscr{C}^{\text {hyp }}$ we would like to have the representation (35). We have

$$
\begin{aligned}
& \psi_{C}\left(b_{1}\right)=u_{1}^{a}(C) v_{11}\left(C_{0}\right)+\varepsilon_{C}\left(b_{1}\right) \varepsilon_{C_{0}}\left(b_{1}\right) e^{j\left[\theta_{C}\left(b_{1}\right)-\theta_{C_{0}}\left(b_{1}\right)\right]} u_{2}^{a}(C) v_{12}\left(C_{0}\right) \\
& \psi_{C}\left(b_{2}\right)=u_{1}^{a}(C) v_{21}\left(C_{0}\right)+\varepsilon_{C}\left(b_{2}\right) \varepsilon_{C_{0}}\left(b_{2}\right) e^{j\left[\theta_{C}\left(b_{2}\right)-\theta_{C_{0}}\left(b_{2}\right)\right]} u_{2}^{a}(C) v_{22}\left(C_{0}\right)
\end{aligned}
$$

Thus to obtain (35) we should have

$$
\varepsilon_{C}\left(b_{1}\right) \varepsilon_{C_{0}}\left(b_{1}\right) e^{j\left[\theta_{C}\left(b_{1}\right)-\theta_{C_{0}}\left(b_{1}\right)\right]}=\varepsilon_{C}\left(b_{2}\right) \varepsilon_{C_{0}}\left(b_{2}\right) e^{j\left[\theta_{C}\left(b_{2}\right)-\theta_{C_{0}}\left(b_{2}\right)\right]}
$$

Thus

$$
\theta_{C}\left(b_{1}\right)-\theta_{C_{0}}\left(b_{1}\right)=\theta_{C}\left(b_{2}\right)-\theta_{C_{0}}\left(b_{2}\right) \text {, or } \theta_{\mathrm{C}}\left(\mathrm{b}_{1}\right)-\theta_{\mathrm{C}}\left(\mathrm{b}_{2}\right)=\theta_{\mathrm{C}_{0}}\left(\mathrm{~b}_{1}\right)-\theta_{\mathrm{C}_{0}}\left(\mathrm{~b}_{2}\right) .
$$

By choosing the representation with (57) we satisfy the above condition.

Theorem 8. We can construct the hyperbolic Hilbert space representation of the contextual Kolmogorov probability model such that the hyperbolic Born's rule holds true for both reference variables a and $b$ iff the matrix of transition probabilities $\mathbf{P}^{b / a}$ is double stochastic.

We remark that by Theorem 5 basic contexts $B_{x}, x \in X$, always belong to $\mathscr{C}^{\text {hyp }}$, so $\psi_{B_{x}} \in H^{\text {hyp }}$; and $B_{x} \in \mathscr{C}^{\text {tr }} \cap \mathscr{C}^{\text {hyp }}$ iff $a$ and $b$ are uniformly distributed ( $\mathbf{P}^{a / b}$ and $\mathbf{P}^{b / a}$ are double stochastic).

\section{Hyperbolic quantum mechanics}

As in the ordinary quantum formalism, we represent physical states by normalized vectors of a hyperbolic Hilbert space $H: \psi \in H$ and $(\psi, \psi)=1$. We shall consider only dichotomous physical variables and quantum states belonging to the two dimensional Hilbert space. Thus everywhere below $H$ denotes the two dimensional space. Let $a=a_{1}, a_{2}$ and $b=b_{1}, b_{2}$ be two physical variables. We represent they by G-linear operators: $\hat{a}=\left|a_{1}\right\rangle\left\langle a_{1}|+| a_{2}\right\rangle\left\langle a_{2}\right|$ and $\hat{b}=\left|b_{1}><b_{1}\right|+\left|b_{2}><b_{2}\right|$, where $\left\{\mid a_{i}>\right\}_{i=1,2}$ and $\left\{\mid b_{i}>\right\}_{i=1,2}$ are two orthonormal bases in $H$. The latter condition plays the fundamental role in hyperbolic quantum mechanics. This is an analogue of the representation of physical observables by self-adjoint operators in the conventional quantum mechanics (in the complex Hilbert space).

Let $\psi$ be a state (normalized vector belonging to $H$ ). We can perform the following operation (which is well defined from the mathematical point of view). We expend the vector $\psi$ with respect to the basis ${ }^{23}\left\{\mid b_{i}>\right\}_{i=1,2}$ :

$$
\psi=v_{1}^{b}\left|b_{1}>+v_{2}^{b}\right| b_{2}>
$$

where the coefficients (coordinates) $v_{i}^{b}$ belong to G. As the basis $\left\{\mid b_{i}>\right\}_{i=1,2}$ is orthonormal, we have (as in the complex case) that:

$$
\left|v_{1}^{b}\right|^{2}+\left|v_{2}^{b}\right|^{2}=1
$$

\footnotetext{
${ }^{23}$ We remark that we consider the two dimensional G-Hilbert space. There exists (by definition) a basis consisting of two vectors.
} 
However, we could not automatically use Born's probabilistic interpretation for normalized vectors in the hyperbolic Hilbert space: it may be that $v_{i}^{b} \notin \mathbf{G}_{+}{ }^{24}$ and hence $\left|v_{i}^{b}\right|^{2}<0$. Since we do not want to consider negative probabilities (cf. [50]), in such a case we cannot use the hyperbolic version of Born's probability interpretation.

Definition 5. A state $\psi$ is decomposable with respect to the system of states $\left\{\left|b_{i}\right\rangle\right\}_{i=1,2}$ (b-decomposable) if

$$
v_{i}^{b} \in \mathbf{G}_{+} .
$$

In such a case we can use generalization of Born's probabilistic interpretation for a hyperbolic Hilbert space (this is a postulate!). Numbers

$$
p_{\psi}^{b}\left(b_{i}\right)=\left|v_{i}^{b}\right|^{2}, i=1,2,
$$

are interpreted as probabilities for values $b=b_{i}$ for the $\mathbf{G}$-quantum state $\psi$.

Thus decomposability is not a mathematical notion. This is not just linear algebraic decomposition of a vector with respect a basis. This is a physical notion describing the possibility of probability interpretation of a measurement over a state. As it was already mentioned, in hyperbolic quantum mechanics a state $\psi \in \mathbf{E}$ is not always decomposable. Thus for an observable $b$ there can exist a state $\psi$ such that the probabilities $p_{\psi}^{b}\left(b_{i}\right)$ are not well defined. One of reasons for this can be the impossibility to perform the $b$-measurement for systems in the state $\psi$. Such a situation is quite natural from the experimental viewpoint. Moreover, it looks surprising that in ordinary quantum (as well as classical) theory we can measure any observable in any state. I think that this is just a consequence of the fact that there was fixed the set of states corresponding to a rather special class of physical observables. Thus in the hyperbolic quantum formalism for each state $\psi \in \mathbf{E}$ there exists its own set of observables $\mathscr{O}(\psi)$. And in general $\mathscr{O}(\psi) \neq \mathscr{O}(\psi)$. We cannot exclude another possibility. The set of observables $\mathscr{O}$ does not depend on a state $\psi$. And the result of an individual measurement of any $b \in \mathscr{O}$ is well defined for any state $\psi$. But relative frequencies of realizations of the value $b=b_{k}$ do not converge to any limit. Therefore probabilities are not well defined. Thus the principle of the statistical stabilization is violated, see [50] for details.

Remark. Let $\mathscr{K}$ be a Kolmogorov probability model and let $\psi \in S_{\mathscr{C} \text { hyp }}$. Thus $\psi=\psi_{C}$ for some $\operatorname{context} C \in \mathscr{C}^{\text {hyp }}$. Let the matrix of transition probabilities $\mathbf{P}^{b / a}$ be double stochastic. Then $\psi$ is decomposable with respect to both reference variables $b$ and $a$. Moreover, basis vectors $e_{i}^{b}=\mid b_{i}>$ are $a$-decomposable and vice versa.

Suppose that a state $\psi \in \mathbf{E}$ is $a$-decomposable:

$$
\psi=v_{1}^{a}\left|a_{1}>+v_{2}^{a}\right| a_{2}>
$$

and the coefficients $v_{i}^{a} \in \mathbf{G}_{+}$.

We also suppose that each state $\mid a_{i}>$ is decomposable with respect to the system of states $\left\{\mid b_{i}>\right\}_{i=1,2}$. We have:

$$
\left|a_{1}>=v_{11}\right| b_{1}>+v_{12}\left|b_{2}>,\right| a_{2}>=v_{21}\left|b_{1}>+v_{22}\right| b_{2}>\text {, }
$$

where the coefficients $v_{i k}$ belong to $\mathbf{G}_{+}$. We have (since both bases are orthonormal):

$$
\left|v_{11}\right|^{2}+\left|v_{12}\right|^{2}=1,\left|v_{21}\right|^{2}+\left|v_{22}\right|^{2}=1
$$

cf. (55). We can use the probabilistic interpretation of numbers $p_{i k}=\left|v_{i k}\right|^{2}$, namely $p_{i k}=p_{\mid a_{i}>}\left(b_{k}\right)$ is the probability for $b=b_{k}$ in the state $\left|a_{i}\right\rangle$.

Let us consider matrix $V=\left(v_{i k}\right)$. As in the complex case, the matrix $V$ is unitary, since vectors $\mid a_{1}>=\left(v_{11}, v_{12}\right)$ and $\left|a_{2}\right\rangle=\left(v_{21}, v_{22}\right)$ are orthonormal. Hence we have normalization conditions (64) and the orthogonality condition:

$$
v_{11} \bar{v}_{21}+v_{12} \bar{v}_{22}=0
$$

cf. (54). It must be noticed that in general unitarity does not imply that $v_{i k} \in \mathbf{G}_{+}$. The latter condition is the additional constraint on the unitary matrix $V$. Let us consider the matrix $\mathbf{P}^{b / a}=\left(p_{i k}\right)$. This matrix is double stochastic (since $V$ is unitary).

By using the G-linear space calculation (the change of the basis) we get $\psi=v_{1}^{b}\left|b_{1}>+v_{2}^{b}\right| b_{2}>$, where $v_{1}^{b}=$ $v_{1}^{a} v_{11}+v_{2}^{a} v_{21}$ and $v_{2}^{b}=v_{1}^{a} v_{12}+v_{2}^{a} v_{22}$.

${ }^{24}$ In fact, in the complex case we have $\mathbf{C}=\mathbf{C}_{+}$; thus there is no problem with positivity. 
We remark that decomposability is not transitive. In principle $\psi$ may be not decomposable with respect to $\left\{\mid b_{i}>\right.$ \}$_{i=1,2}$, despite the decomposability of $\psi$ with respect to $\left\{\mid a_{i}>\right\}_{i=1,2}$ and the decomposability of the latter system with respect to $\left\{\mid b_{i}>\right\}_{i=1,2}$.

The possibility of decomposability is based on two (totally different) conditions: (61), normalization, and (62), positivity. Any G-unitary transformation preserves the normalization condition. Thus we get automatically that $\left|v_{1}^{b}\right|^{2}+$ $\left|v_{2}^{b}\right|^{2}=1$. However, the condition of positivity in general is not preserved: it can be that $v_{i}^{b} \notin \mathbf{G}_{+}$even if we have $v_{i}^{a} \in \mathbf{G}_{+}$and the matrix $V$ is $\mathbf{G}$-unitary.

Finally, suppose that $\psi$ is decomposable with respect to $\left\{\mid b_{i}>\right\}_{i=1,2}$. Thus $v_{k}^{b} \in \mathbf{G}_{+}$. Therefore coefficients $p_{\psi}^{b}\left(b_{i}\right)=\left|v_{i}^{b}\right|^{2}$ can be interpreted as probabilities for $b=b_{k}$ for the G-quantum state $\psi$.

Let us consider states such that coefficients $v_{i}^{a}, v_{i k}$ belong to $\mathbf{G}_{+}^{*}$. We can uniquely represent them as $v_{i}^{a}= \pm \sqrt{p_{\psi}^{a}\left(a_{i}\right)} e^{j \xi_{i}}, v_{i k}= \pm \sqrt{p_{i k}} e^{j \gamma_{i k}}, i, k,=1,2$.

We find that

$$
\begin{aligned}
& p_{\psi}^{b}\left(b_{1}\right)=p_{\psi}^{a}\left(a_{1}\right) p_{11}+p_{\psi}^{a}\left(a_{2}\right) p_{21}+2 \varepsilon_{1} \cosh \theta_{1} \sqrt{p_{\psi}^{a}\left(a_{1}\right) p_{11} p_{\psi}^{a}\left(a_{2}\right) p_{21}} \\
& p_{\psi}^{b}\left(b_{2}\right)=p_{\psi}^{a}\left(a_{1}\right) p_{12}+p_{\psi}^{a}\left(a_{2}\right) p_{22}+2 \varepsilon_{2} \cosh \theta_{2} \sqrt{p_{\psi}^{a}\left(a_{1}\right) p_{12} p_{\psi}^{a}\left(a_{2}\right) p_{22}},
\end{aligned}
$$

where $\theta_{i}=\eta+\gamma_{i}$ and $\eta=\xi_{1}-\xi_{2}, \gamma_{1}=\gamma_{11}-\gamma_{21}, \gamma_{1}=\gamma_{12}-\gamma_{22}$ and $\varepsilon_{i}= \pm$. To find the right relation between signs of the last terms in equations (66), (67), we use the normalization condition

$$
\left|v_{2}^{b}\right|^{2}+\left|v_{2}^{b}\right|^{2}=1
$$

(which is a consequence of the normalization of $\psi$ and orthonormality of the system $\left\{\mid b_{i}>\right\}_{i=1,2}$ ). ${ }^{25}$

Equation (68) is equivalent to the equation:

$$
\sqrt{p_{12} p_{22}} \cosh \theta_{2} \pm \sqrt{p_{11} p_{21}} \cosh \theta_{2}=0 .
$$

Thus we have to choose opposite signs in equations (66), (67). Unitarity of $V$ also implies that $\theta_{1}-\theta_{2}=0$, so $\gamma_{1}=\gamma_{2}$. We recall that in the ordinary quantum mechanics we have similar conditions, but trigonometric functions are used instead of hyperbolic and phases $\gamma_{1}$ and $\gamma_{2}$ are such that $\gamma_{1}-\gamma_{2}=\pi$.

Finally, we get that unitary linear transformations in the G-Hilbert space (in the domain of decomposable states) represent the following transformation of probabilities:

$$
\begin{aligned}
& p_{\psi}^{b}\left(b_{1}\right)=p_{\psi}^{a}\left(a_{1}\right) p_{11}+p_{\psi}^{a}\left(a_{2}\right) p_{21} \pm 2 \varepsilon_{1} \cosh \theta_{1} \sqrt{p_{\psi}^{a}\left(a_{1}\right) p_{11} p_{\psi}^{a}\left(a_{2}\right) p_{21}} \\
& p_{\psi}^{b}\left(b_{2}\right)=p_{\psi}^{a}\left(a_{1}\right) p_{12}+p_{\psi}^{a}\left(a_{2}\right) p_{22} \mp 2 \varepsilon_{2} \cosh \theta_{2} \sqrt{p_{\psi}^{a}\left(a_{1}\right) p_{12} p_{\psi}^{a}\left(a_{2}\right) p_{22}}
\end{aligned}
$$

This is a kind of hyperbolic interference.

\section{COMPLEX AMPLITUDES OF PROBABILITIES IN THE CASE OF MULTIVARIED REFERENCE VARIABLES}

The general case of random variables taking $n \geq 2$ different values can be (inductively) reduced to the case of dichotomous random variables (cf., e.g., Mackey [14] who also reduced the study of arbitrary observables to the study of dichotomous variables - questions). We consider two incompatible random variables taking $n$ values: $b=b_{1}, \ldots, b_{n}$ and $a=a_{1}, \ldots, a_{n}$.

Lemma 7. Let $B, C, D_{1}, D_{2} \in \mathscr{F}, \mathbf{P}(C) \neq 0$ and $D_{1} \cap D_{2}=\emptyset$. Then

$$
\mathbf{P}\left(B\left(D_{1} \cup D_{2}\right) / C\right)=\mathbf{P}\left(B D_{1} / C\right)+\mathbf{P}\left(B D_{2} / C\right)
$$

\footnotetext{
${ }^{25}$ We remark that the normalization condition (68) can be reduced to relations between coefficients of the transition matrix $V$. So it does not depend on the original $a$-decomposition of $\psi$, namely coefficients $v_{i}^{a}$. Condition of positivity, $\left|v_{i}^{b}\right|^{2} \geq 0$, could not be written by using only coefficients of $V$. We also need to use coefficients $v_{i}^{a}$. Therefore it seems to be impossible to find such a class of linear transformations $V$ that would preserve condition of positivity, "decomposition-group" of operators.
} 
Proposition 5. (The formula of total probability) Let conditions of Lemma 7 hold and let $\mathbf{P}\left(D_{j} C\right) \neq 0$. Then

$$
\mathbf{P}\left(B\left(D_{1} \cup D_{2}\right) / C\right)=\mathbf{P}\left(B / D_{1} C\right) \mathbf{P}\left(D_{1} / C\right)+\mathbf{P}\left(B / D_{2} C\right) \mathbf{P}\left(D_{2} / C\right)
$$

Proposition 6. (Contextual formula of total probability) Let conditions of Proposition 5 hold true and let $\mathbf{P}\left(B D_{j}\right) \neq$ $0, j=1,2$. Then

$$
\begin{gathered}
\mathbf{P}\left(B\left(D_{1} \cup D_{2}\right) / C\right)=\mathbf{P}\left(B / D_{1}\right) \mathbf{P}\left(D_{1} / C\right)+\mathbf{P}\left(B / D_{2}\right) \mathbf{P}\left(D_{2} / C\right)+ \\
2 \lambda\left(B /\left\{D_{1}, D_{2}\right\}, C\right) \sqrt{\mathbf{P}\left(B / D_{1}\right) \mathbf{P}\left(D_{1} / C\right) \mathbf{P}\left(B / D_{2}\right) \mathbf{P}\left(D_{2} / C\right)},
\end{gathered}
$$

where

$$
\lambda\left(B /\left\{D_{1}, D_{2}\right\}, C\right)=\frac{\delta\left(B /\left\{D_{1}, D_{2}\right\}, C\right)}{2 \sqrt{\mathbf{P}\left(B / D_{1}\right) \mathbf{P}\left(D_{1} / C\right) \mathbf{P}\left(B / D_{2}\right) \mathbf{P}\left(D_{2} / C\right)}}
$$

and

$$
\begin{gathered}
\delta\left(B /\left\{D_{1}, D_{2}\right\}, C\right)=\mathbf{P}\left(B\left(D_{1} \cup D_{2}\right) / C\right)-\sum_{j=1}^{2} \mathbf{P}\left(B / D_{j}\right) \mathbf{P}\left(D_{j} / C\right) \\
=\sum_{j=1}^{2} \mathbf{P}\left(D_{j} / C\right)\left(\mathbf{P}\left(B / D_{j} C\right)-\mathbf{P}\left(B / D_{j}\right)\right)
\end{gathered}
$$

In the construction of a Hilbert space representation of contexts for multivalued observables there will be used the following combination of formulas (72) and (74).

Lemma 8. Let conditions of Lemma 7 hold and let $\mathbf{P}\left(B D_{1}\right), \mathbf{P}\left(C D_{1}\right)$ and $\mathbf{P}\left(B D_{2} C\right)$ be strictly positive. Then

$$
\begin{aligned}
& \mathbf{P}\left(B\left(D_{1} \cup D_{2}\right) / C\right)=\mathbf{P}\left(B / D_{1}\right) \mathbf{P}\left(D_{1} / C\right)+\mathbf{P}\left(B D_{2} / C\right) \\
& +2 \mu\left(B /\left\{D_{1}, D_{2}\right\}, C\right) \sqrt{\mathbf{P}\left(B / D_{1}\right) \mathbf{P}\left(D_{1} / C\right) \mathbf{P}\left(B D_{2} / C\right)}
\end{aligned}
$$

where $\mu\left(B /\left\{D_{1}, D_{2}\right\}, C\right)=\frac{\mathbf{P}\left(B\left(D_{1} \cup D_{2}\right) / C\right)-\mathbf{P}\left(B / D_{1}\right) \mathbf{P}\left(D_{1} / C\right)-\mathbf{P}\left(B D_{2} / C\right)}{2 \sqrt{\mathbf{P}\left(B / D_{1}\right) \mathbf{P}\left(D_{1} / C\right) \mathbf{P}\left(B D_{2} / C\right)}}$

Suppose that coefficients of statistical disturbance $\mu$ and $\lambda$ are bounded by 1 . Then we can represent them in the trigonometric form:

$$
\begin{gathered}
\lambda\left(B /\left\{D_{1}, D_{2}\right\}, C\right)=\cos \theta\left(B /\left\{D_{1}, D_{2}\right\}, C\right) \\
\mu\left(B /\left\{D_{1}, D_{2}\right\}, C\right)=\cos \gamma\left(B /\left\{D_{1}, D_{2}\right\}, C\right)
\end{gathered}
$$

By inserting these cos-expressions in (74) and (76) we obtain trigonometric transformations of probabilities. We have (by Lemma 8):

$$
\begin{gathered}
\mathbf{P}\left(B_{x} / C\right)=\mathbf{P}\left(B_{x}\left(A_{1} \cup \ldots \cup A_{n}\right) / C\right) \\
=\mathbf{P}\left(B_{x} / A_{1}\right) \mathbf{P}\left(A_{1} / C\right)+\mathbf{P}\left(B_{x}\left(A_{2} \cup \ldots \cup A_{n}\right) / C\right) \\
+2 \mu\left(B_{x} /\left\{A_{1}, A_{2} \cup \ldots \cup A_{n}\right\}, C\right) \sqrt{\mathbf{P}\left(B_{x} / A_{1}\right) \mathbf{P}\left(A_{1} / C\right) \mathbf{P}\left(B_{x}\left(A_{2} \cup \ldots \cup A_{n}\right) / C\right)}
\end{gathered}
$$

where

$$
\begin{gathered}
\mu\left(B_{x} /\left\{A_{1}, A_{2} \cup \ldots \cup A_{n}\right\}, C\right) \\
=\frac{\mathbf{P}\left(B_{x}\left(A_{1} \cup \ldots \cup A_{n}\right) / C\right)-\mathbf{P}\left(B_{x} / A_{1}\right) \mathbf{P}\left(A_{1} / C\right)-\mathbf{P}\left(B_{x}\left(A_{2} \cup \ldots \cup A_{n}\right) / C\right)}{2 \sqrt{\left.\mathbf{P}\left(B_{x} / A_{1}\right) \mathbf{P}\left(A_{1} / C\right) \mathbf{P}\left(B_{x}\left(A_{2} \cup \ldots \cup A_{n}\right) / C\right)\right)}} .
\end{gathered}
$$

Suppose that the coefficients of statistical disturbance are relatively small for all $x \in X:\left|\mu\left(B_{x} /\left\{A_{1}, A_{2} \cup \ldots \cup A_{n}\right\}, C\right)\right| \leq$ 1. Then we can represent these coefficients as

$$
\mu\left(B_{x} /\left\{A_{1}, A_{2} \cup \ldots \cup A_{n}\right\}, C\right)=\cos \gamma\left(B_{x} /\left\{A_{1}, A_{2} \cup \ldots \cup A_{n}\right\}, C\right) .
$$

Thus the probability $\mathbf{P}\left(B_{x} / C\right) \equiv \mathbf{P}\left(B_{x}\left(A_{1} \cup \ldots \cup A_{n}\right) / C\right)$ can be represented as the square of the absolute value of the complex amplitude:

$$
\psi_{C}(x) \equiv \psi_{C}^{(1)}(x)=\sqrt{\mathbf{P}\left(B_{x} / A_{1}\right) \mathbf{P}\left(A_{1} / C\right)}+e^{i \gamma_{C}^{(1)}(x)} \sqrt{\mathbf{P}\left(B_{x}\left(A_{2} \cup \ldots \cup A_{n}\right) / C\right)},
$$


where the phase $\gamma_{C}^{(1)}(x) \equiv \gamma\left(B_{x} /\left\{A_{1}, A_{2} \cup \ldots \cup A_{n}\right\}, C\right)$. In the same way the probability in the second summand can be represented as:

$$
\begin{gathered}
\mathbf{P}\left(B_{x}\left(A_{2} \cup \ldots \cup A_{n}\right) / C\right)=\mathbf{P}\left(B_{x} / A_{2}\right) \mathbf{P}\left(A_{2} / C\right)+\mathbf{P}\left(B_{x}\left(A_{3} \cup \ldots \cup A_{n}\right) / C\right)+ \\
2 \mu\left(B_{x} /\left\{A_{2}, A_{3} \cup \ldots \cup A_{n}\right\}, C\right) \sqrt{\mathbf{P}\left(B_{x} / A_{2}\right) \mathbf{P}\left(A_{2} / C\right) \mathbf{P}\left(B_{x}\left(A_{3} \cup \ldots \cup A_{n}\right) / C\right)},
\end{gathered}
$$

where

$$
\begin{gathered}
\mu\left(B_{x} /\left\{A_{2}, A_{3} \cup \ldots \cup A_{n}\right\}, C\right) \\
=\frac{\mathbf{P}\left(B_{x}\left(A_{2} \cup \ldots \cup A_{n}\right) / C\right)-\mathbf{P}\left(B_{x} / A_{2}\right) \mathbf{P}\left(A_{2} / C\right)-\mathbf{P}\left(B_{x}\left(A_{3} \cup \ldots \cup A_{n}\right) / C\right)}{2 \sqrt{\mathbf{P}\left(B_{x} / A_{2}\right) \mathbf{P}\left(A_{2} / C\right) \mathbf{P}\left(B_{x}\left(A_{3} \cup \ldots \cup A_{n}\right) / C\right)}} .
\end{gathered}
$$

By supposing that these coefficients of statistical disturbance are bounded by 1 we represent the probability as the square of the absolute value of the complex amplitude:

$$
\psi_{C}^{(2)}(x)=\sqrt{\mathbf{P}\left(B_{x} / A_{2}\right) \mathbf{P}\left(A_{2} / C\right)}+e^{i \gamma_{C}^{(2)}(x)} \sqrt{\mathbf{P}\left(B_{x}\left(A_{3} \cup \ldots \cup A_{n}\right) / C\right)},
$$

where $\gamma_{C}^{(2)}(x)= \pm \arccos \mu\left(B_{x} /\left\{A_{2}, A_{3}, \cup \ldots \cup A_{n}\right\}, C\right)$. On the $j t h$ step we represent $\mathbf{P}\left(B_{x}\left(A_{j} \cup \ldots \cup A_{n}\right) / C\right)$ as the square of the absolute value of the complex amplitude

$$
\psi_{C}^{(j)}(x)=\sqrt{\mathbf{P}\left(B_{x} / A_{j}\right) \mathbf{P}\left(A_{j} / C\right)}+e^{i \gamma_{C}^{(j)}(x)} \sqrt{\mathbf{P}\left(B_{x}\left(A_{j+1} \cup \ldots \cup A_{n}\right) / C\right)},
$$

where $\gamma_{C}^{(j)}(x)$ is the phase of the coefficient

$$
\begin{gathered}
\mu\left(B_{x} /\left\{A_{j}, A_{j+1} \cup \ldots \cup A_{n}\right\}, C\right) \\
=\frac{\mathbf{P}\left(B_{x}\left(A_{j} \cup \ldots \cup A_{n}\right) / C\right)-\mathbf{P}\left(B_{x} / A_{j}\right) \mathbf{P}\left(A_{j} / C\right)-\mathbf{P}\left(B_{x}\left(A_{j+1} \cup \ldots \cup A_{n}\right) / C\right)}{2 \sqrt{\mathbf{P}\left(B_{x} / A_{j}\right) \mathbf{P}\left(A_{j} / C\right) \mathbf{P}\left(B_{x}\left(A_{j+1} \cup \ldots \cup A_{n}\right) / C\right)}} .
\end{gathered}
$$

It is supposed that at each step we obtain coefficients $|\mu|$ bounded by 1 . At the step $j=n-1$ we should represent the probability $\mathbf{P}\left(B_{x}\left(A_{n-1} \cup A_{n}\right) / C\right)$. Here we can already totally eliminate the $C$-contextuality for $B_{x}$ :

$$
\begin{aligned}
& \mathbf{P}\left(B_{x}\left(A_{n-1} \cup A_{n}\right) / C\right)=\mathbf{P}\left(B_{x} / A_{n-1}\right) \mathbf{P}\left(A_{n-1} / C\right)+\mathbf{P}\left(B_{x} / A_{n}\right) \mathbf{P}\left(A_{n} / C\right) \\
& +2 \lambda\left(B_{x} /\left\{A_{n-1}, A_{n}\right\}\right) \sqrt{\mathbf{P}\left(B_{x} / A_{n-1}\right) \mathbf{P}\left(A_{n-1} / C\right) \mathbf{P}\left(B_{x} / A_{n}\right) \mathbf{P}\left(A_{n} / C\right),}
\end{aligned}
$$

where the coefficient of statistical disturbance $\lambda$ was defined by (75). And if $|\lambda|$ is bounded by 1 then we can represent the probability as the square of the absolute value of the complex amplitude:

$$
\psi_{C}^{(n-1)}(x)=\sqrt{\mathbf{P}\left(B_{x} / A_{n-1}\right) \mathbf{P}\left(A_{n-1} / C\right)}+e^{i \theta_{C}(x)} \sqrt{\mathbf{P}\left(B_{x} / A_{n}\right) \mathbf{P}\left(A_{n} / C\right)},
$$

where $\theta_{C}(x)= \pm \arccos \lambda\left(x /\left\{A_{n-1}, A_{n}\right\}, C\right)$.

We have:

$$
\psi_{C}^{(j)}(x)=\sqrt{\mathbf{P}\left(B_{x}\left(A_{j} \cup \ldots \cup A_{n}\right) / C\right)} e^{i \alpha_{C}^{(j)}(x)},
$$

where $\alpha_{C}^{(j)}(x)=\arg \psi_{C}^{(j)}(x)=\arccos \frac{M_{j}}{N_{j}}$, where $M_{j}=\sqrt{\mathbf{P}\left(B_{x} / A_{j}\right) \mathbf{P}\left(A_{j} / C\right)}$

$+\mu\left(B_{x} /\left\{A_{j}, A_{j+1} \cup \ldots \cup A_{n}\right\}, C\right) \sqrt{\mathbf{P}\left(B_{x}\left(A_{j+1} \cup \ldots \cup A_{n}\right) / C\right)}$,

$N_{j}=\sqrt{\mathbf{P}\left(B_{x}\left(A_{j} \cup \ldots \cup A_{n}\right) / C\right)}$. Finally, we have:

$$
\begin{gathered}
\alpha_{C}^{(n-1)}(x)=\arg \psi_{C}^{(n-1)}(x) \\
=\arccos \frac{\sqrt{\mathbf{P}\left(B_{x} / A_{n-1}\right) \mathbf{P}\left(A_{n-1} / C\right)}+\lambda\left(B_{x} /\left\{A_{n-1}, A_{n}\right\}, C\right) \sqrt{\mathbf{P}\left(B_{x} / A_{n}\right) \mathbf{P}\left(A_{n} / C\right)}}{\sqrt{\mathbf{P}\left(B_{x}\left(A_{n-1} \cup A_{n}\right) / C\right)}} .
\end{gathered}
$$

Thus we have:

$$
\psi_{C}(x)=\sqrt{\mathbf{P}\left(B_{x} / A_{1}\right) \mathbf{P}\left(A_{1} / C\right)}+e^{i\left[\gamma_{C}^{(1)}(x)-\alpha_{C}^{(2)}(x)\right]} \psi_{C}^{(2)}(x)
$$




$$
\begin{gathered}
=\sqrt{\mathbf{P}\left(B_{x} / A_{1}\right) \mathbf{P}\left(A_{1} / C\right)}+e^{i \beta_{C}^{(2)}(x)} \sqrt{\mathbf{P}\left(B_{x} / A_{2}\right) \mathbf{P}\left(A_{2} / C\right)} \\
+e^{i \beta_{C}^{(3)}(x)} \psi_{C}^{(3)}(x),
\end{gathered}
$$

where

$$
\beta_{C}^{(2)}(x)=\gamma_{C}^{(1)}(x)-\alpha_{C}^{(2)}(x), \beta_{C}^{(3)}(x)=\beta_{C}^{2}(x)+\gamma_{C}^{(2)}(x)-\alpha_{C}^{(3)}(x)
$$

Finally, we obtain:

$$
\psi_{C}(x)=\sum_{j=1}^{n} e^{i \beta_{C}^{(j)}(x)} \sqrt{\mathbf{P}\left(B_{x} / A_{j}\right) \mathbf{P}\left(A_{j} / C\right)}
$$

with $\beta_{C}^{(1)}(x)=0$ (this is just due to our special choice of a representation) and $\beta_{C}^{(n)}(x)=\beta_{C}^{(n-1)}(x)+\theta_{C}(x)$.

Thus by inductive splitting of multivalued variables into dichotomous variables we represented contextual probabilities by complex amplitudes $\psi_{C}(x)$.

By using the standard in this paper symbols $p(x / y)=\mathbf{P}\left(B_{x} / A_{y}\right)$ and $p_{C}^{b}(x)=\mathbf{P}\left(B_{x} / C\right), p_{C}^{a}(y)=\mathbf{P}\left(A_{y} / C\right)$ we write

$$
\psi_{C}(x)=\sum_{y} e^{i \beta_{C}^{(y)}(x)} \sqrt{p_{C}^{a}(y) p(x / y)}
$$

In particular, for $n=3$ we have

$$
\psi_{C}(x)=\sqrt{p_{C}^{a}\left(a_{1}\right) p\left(x / a_{1}\right)}+e^{i \beta_{C}^{(2)}(x)} \sqrt{p_{C}^{a}\left(a_{2}\right) p\left(x / a_{2}\right)},+e^{i \beta_{C}^{(3)}(x)} \sqrt{p_{C}^{a}\left(a_{3}\right) p\left(x / a_{3}\right)},
$$

where

$$
\beta_{C}^{(2)}(x)=\gamma_{C}^{(1)}(x)-\alpha_{C}^{(2)}(x), \beta_{C}^{(3)}(x)=\beta_{C}^{(2)}(x)+\theta_{C}(x) .
$$

We remark that each phase $\beta_{C}^{(j)}(x)$ depends on all three $a$-contexts, $A_{1}, A_{2}, A_{3}$. So we cannot use the symbol $\beta_{C}(x / y)$. In $\beta_{C}^{(y)}(x)$ the $y$ is just the summation index; in fact, $\beta_{C}^{(y)}(x) \equiv \beta_{C}^{(y)}\left(x / A_{1}, A_{2}, A_{3}\right)$. We remark that the probability $p_{C}^{b}(x)$ can be represented as

$$
\begin{gathered}
p_{C}^{b}(x)=\left|\psi_{C}(x)\right|^{2}=\sum_{y} p_{C}^{a}(y) p(x / y) \\
+2 \sum_{y_{1}<y_{2}} \cos \left[\beta_{C}^{\left(y_{2}\right)}(x)-\beta_{C}^{\left(y_{1}\right)}(x)\right] \sqrt{p_{C}^{a}\left(y_{1}\right) p_{C}^{a}\left(y_{2}\right) p\left(x / y_{1}\right) p\left(x / y_{2}\right)} .
\end{gathered}
$$

We can proceed in the same way as in the case of dichotomous random variables.

I would like to thank L. Ballentine, S. Gudder, A. Holevo, P. Lahti, B Hiley, C. Fuchs, A. Peres, I. Volovich, R. Gill, K. Hess, W. Philipp, L. Accardi, A. Aspect, G. 't Hooft, J. Bub, T. Maudlin, H. Rauch, G. Emch, V. Belavkin, A. Leggett, I. Helland, P. Kwait, G. Adenier, for discussions on probabilistic foundations of quantum theory. This paper was partially supported by EU-Network "QP and Applications" and Nat. Sc. Found., grant N PHY99-07949 at KITP, Santa-Barbara, visiting professor fellowship at Russian State Humanitarian University and the Profile Mathematical Modeling of Växjö University.

\section{APPENDIX ON INCOMPATIBLE RANDOM VARIABLES}

Proposition 7. Let $\left\{A_{j}\right\}$ and $\left\{B_{k}\right\}$ be two families of subsets of some set $\Omega$ and $\Omega=\cup_{j} A_{j}=\cup_{k} B_{k}$ and let

$$
A_{j} B_{k} \neq \emptyset
$$

for any pair $(j, k)$. Then

$$
\text { Neither } A_{j} \subset B_{k} \text { nor } B_{k} \subset A_{j}
$$

for any pair $(j, k)$. If $n=2$ then conditions (77) and (78) are equivalent.

Proof. Let (77) hold true. Suppose that there exists $(j, k)$ such that $A_{j} \subset B_{k}$. Thus we should have $A_{j} B_{i}=\emptyset$ for any $i \neq k$. Let (78) hold true and let $n=2: \mathscr{A}=\left\{A_{1}, A_{2}=\Omega \backslash A_{1}\right\}$ and $\mathscr{B}=\left\{B_{1}, B_{2}=\Omega \backslash B_{1}\right\}$. Suppose that, e.g., $A_{1} B_{1}=\emptyset$. Then we should have $A_{1} \subset B_{2}$. 
If $n \neq 3$ then in general the condition (78) does not imply the condition (77). We can consider the following example. Let $\Omega=\left\{\omega_{1}, \ldots, \omega_{7}\right\}$ and let $A_{1}=\left\{\omega_{1}, \omega_{2} \omega_{3}\right\}, A_{2}=\left\{\omega_{4}, \omega_{5}\right\}, A_{3}=\left\{\omega_{6}, \omega_{7}\right\}$ and $B_{1}=\left\{\omega_{1}, \omega_{4}\right\}, B_{2}=$ $\left\{\omega_{2}, \omega_{5}, \omega_{6}\right\}, B_{3}=\left\{\omega_{3}, \omega_{7}\right\}$. Here (78) holds true but $A_{2} B_{3} \neq \emptyset$.

\section{REFERENCES}

1. A. N. Kolmogoroff, Grundbegriffe der Wahrscheinlichkeitsrechnung, Springer Verlag, Berlin, 1933; reprinted: Foundations of the Probability Theory, Chelsea Publ. Comp., New York, 1956.

2. D. Hilbert, J. von Neumann, L. Nordheim, Math. Ann., 98, 1-30 (1927).

3. P. A. M. Dirac, The Principles of Quantum Mechanics, Oxford Univ. Press, 1930.

4. W. Heisenberg, Physical principles of quantum theory, Chicago Univ. Press, 1930.

5. J. von Neumann, Mathematical foundations of quantum mechanics, Princeton Univ. Press, Princeton, N.J., 1955.

6. A. Einstein, B. Podolsky, N. Rosen, Phys. Rev. 47, 777-780 (1935).

7. N. Bohr, Phys. Rev. 48, 696-702 (1935).

8. D. Bohm, Quantum theory, Prentice-Hall, Englewood Cliffs, New-Jersey, 1951.

9. A. Lande, Foundations of quantum theory, Yale Univ. Press, 1955.

10. A. Lande, New foundations of quantum mechanics, Cambridge Univ. Press, Cambridge, 1965.

11. A. S. Wightman, Hilbert's sixth problem: mathematical treatment of the axioms of physics, Proc. Symposia in Pure Math., 28, 147-233 (1976).

12. L. De Broglie, The current interpretation of wave mechanics, critical study. Elsevier Publ., Amsterdam-LondonNew York, 1964.

13. J. S. Bell, Speakable and unspeakable in quantum mechanics, Cambridge Univ. Press, 1987.

14. G. W. Mackey, Mathematical foundations of quantum mechanics, W. A. Benjamin INc, New York, 1963.

15. S. Kochen and E. Specker, J. Math. Mech., 17, 59-87 (1967).

16. L. E. Ballentine, Rev. Mod. Phys., 42, 358-381 (1970).

17. G. Ludwig, Foundations of quantum mechanics, Springer, Berlin, 1983.

18. E. B. Davies, J. T. Lewis, Comm. Math. Phys. 17, 239-260 (1970).

19. E. Nelson, Quantum fluctuation, Princeton Univ. Press, Princeton, 1985.

20. D. Bohm and B. Hiley, The undivided universe: an ontological interpretation of quantum mechanics, Routledge and Kegan Paul, London, 1993.

21. S. P. Gudder, Trans. AMS 119, 428-442 (1965).

22. S. P. Gudder, Axiomatic quantum mechanics and generalized probability theory, Academic Press, New York, 1970.

23. S. P. Gudder, "An approach to quantum probability" in Foundations of Probability and Physics, edited by A.

Yu. Khrennikov, Quantum Prob. White Noise Anal., 13, WSP, Singapore, 2001, pp. 147-160.

24. R. Feynman and A. Hibbs, Quantum Mechanics and Path Integrals, McGraw-Hill, New-York, 1965.

25. J. M. Jauch, Foundations of Quantum Mechanics, Addison-Wesley, Reading, Mass., 1968.

26. A. Peres, Quantum Theory: Concepts and Methods, Dordrecht, Kluwer Academic, 1994.

27. L. Accardi, "The probabilistic roots of the quantum mechanical paradoxes" in The wave-particle dualism. A

tribute to Louis de Broglie on his 90th Birthday, edited by S. Diner, D. Fargue, G. Lochak and F. Selleri, D. Reidel Publ. Company, Dordrecht, 1984, pp. 297-330.

28. L. Accardi, Urne e Camaleoni: Dialogo sulla realta, le leggi del caso e la teoria quantistica, Il Saggiatore, Rome, 1997.

29. L. E. Ballentine, Quantum mechanics, Englewood Cliffs, New Jersey, 1989.

30. L. E. Ballentine, "Interpretations of probability and quantum theory", in Foundations of Probability and Physics, edited by A. Yu. Khrennikov, Q. Prob. White Noise Anal., 13, WSP, Singapore, 2001, pp. 71-84.

31. A. S. Holevo, Probabilistic and statistical aspects of quantum theory, North-Holland, Amsterdam, 1982.

32. A. S. Holevo, Statistical structure of quantum theory, Springer, Berlin-Heidelberg, 2001.

33. P. Busch, M. Grabowski, P. Lahti, Operational Quantum Physics, Springer Verlag,Berlin, 1995.

34. A. Yu. Khrennikov (editor), Foundations of Probability and Physics, Q. Prob. White Noise Anal., 13, WSP, Singapore, 2001.

35. A. Yu. Khrennikov (editor), Quantum Theory: Reconsideration of Foundations, Ser. Math. Modeling, 2, Växjö Univ. Press, 2002.

36. A. Yu. Khrennikov (editor), Foundations of Probability and Physics-2, Ser. Math. Modeling, 5, Växjö Univ. Press, 2003. 
37. A. Yu. Khrennikov (editor), Quantum Theory: Reconsideration of Foundations-2, Ser. Math. Modeling, 10, Växjö Univ. Press, 2004.

38. A. Yu. Khrennikov, J. Phys.A: Math. Gen. 34, 9965-9981 (2001).

39. A . Yu. Khrennikov, Il Nuovo Cimento B 117, 267-281 (2002).

40. A. Yu. Khrennikov, J. Math. Phys. 43, 789-802 (2002).

41. A. Yu. Khrennikov, Information dynamics in cognitive, psychological and anomalous phenomena, Ser. Fundamental Theories of Physics, Kluwer, Dordreht, 2004.

42. A. Yu. Khrennikov, J. Math. Phys. 44, 2471- 2478 (2003).

43. A. Yu. Khrennikov, Phys. Lett. A 316, 279-296 (2003).

44. A. Yu. Khrennikov, Advances in Applied Clifford Algebras 13(1), 1-9 (2003).

45. A. Yu. Khrennikov, Annalen der Physik 12, 575-585 (2003).

46. A. N. Shiryayev, Probability, Springer, New York-Berlin-Heidelberg, 1991.

47. E. Conte, O. Todarello, A. Federici, F. Vitiello, M. Lopane, A. Yu. Khrennikov, "A preliminary evidence of quantum-like behaviour in measurements of mental states" in Quantum Theory: Reconsideration of Foundations, edited by A. Yu. Khrennikov, Ser. Math. Modeling, 10, Växjö Univ. Press, 2004, pp. 679-702.

48. H. Atmanspacher, H. Primas, "Epistemic and ontic quantum realities", in Foundations of Probability and Physics-3, edited by A. Yu. Khrennikov, AIP Conference Proceedings, 2005.

49. A. Yu. Khrennikov, Supernalysis, Nauka, Fizmatlit, Moscow, 1997 (in Russian). English translation: Kluwer, Dordreht, 1999.

50. A. Yu. Khrennikov, Interpretations of Probability, VSP Int. Sc. Publishers, Utrecht/Tokyo, 1999 (second edition, 2004). 